\title{
Diffraction of hydroelastic waves by multiple vertical circular cylinders
}

\author{
K. Ren • G. X. Wu • C. Y. Ji
}

Received: 3 May 2018 / Accepted: 21 September 2018 / Published online: 10 November 2018

(C) The Author(s) 2018

\begin{abstract}
The diffraction problem of hydroelastic waves beneath an ice sheet by multiple bottom-mounted circular cylinders is considered. The elastic thin-plate theory is adopted to model the ice sheet, while the linearized velocity potential theory adopted for the fluid flow. The velocity potential corresponding to each cylinder is expanded into a series of eigenfunctions, and the total potential is expressed as a summation of these expansions over the entire $N C$ number of cylinders. For each cylinder, the Green's second identity is used outside its domain to obtain a set of linear equations. For each different cylinder, the domain used is different. $N C$ cylinders give $N C$ sets of coupled linear equations. Investigations are made for different arrangements of cylinders, piercing through ice sheets. Results for the wave forces on the cylinders with clamped and free conditions of the ice edge are obtained. Physical phenomena corresponding to cylinders arranged in square, in an array, in a double-array and in a staggered double array are discussed.
\end{abstract}

Keywords Free/clamped edge conditions · Hydroelastic wave diffraction · Ice sheet - Multiple cylinders and cylinder arrays

\section{Introduction}

Vertical circular columns are commonly adopted in ocean engineering as components of coastal and offshore structures, such as bridge pylons and tension leg platforms. The wave forces acting on these columns are usually of major interest. Extensive research has been conducted on the water wave diffraction by vertical cylinders in open water. Havelock [1] considered the diffraction problem of a single vertical circular cylinder in regular wave of infinite depth and derived an exact solution. MacCamy and Fuchs [2] used the method of separation of variables involving Hankel function for a circular cylinder in the finite water depth. For a vertical cylinder with an elliptic cross section, Chen and Mei [3] adopted the variable separation method involving Mathieu functions in the elliptic cylindrical coordinate system. Williams [4] adopted both semi-analytical and numerical methods for the diffraction problem of an elliptic cylinder. The first one was based on elliptic eccentricity, and the Mathieu functions in the

K. Ren · G. X. Wu (凶)

Department of Mechanical Engineering, University College London, Torrington Place, London WC1E 7JE, UK

e-mail: g.wu@ucl.ac.uk

C. Y. Ji

School of Naval Architecture and Ocean Engineering, Jiangsu University of Science and Technology, Zhenjiang 212003, China 
obtained exact solution can be expanded in series of Bessel functions. The second one was a boundary element method based on the Green's theorem; the elliptical contour was divided into small segments where the potential in each segment is assumed to be constant. The wave diffraction problem of uniform vertical cylinders with arbitrary cross section has been investigated numerically by Isaacson [5], Wu and Price [6], Zhu and Moule [7] and Mansour et al. [8]. Recently, semi-analytical methods were also adopted to get solutions of such problems. Liu et al. [9] adopted the method of separation of variables using the expression form of potential given by MacCamy and Fuchs [2] for circular cylinder and expanding the radial functions of the body surface as well as Bessel functions into a Fourier series with unknown coefficients. Dişibüyük et al. [10] used an asymptotic approach by defining a small parameter $\varepsilon$ corresponding to the variation of the radius from a circular cylinder and calculating the boundary value problem on the circular surface to obtain a fifth-order asymptotic solution.

In addition to studies on a single cylinder mentioned above, there have been works on multiple vertical cylinders arranged in an array or in a group, as done by Ohkusu [11], Kagemoto and Yue [12], Linton and Evans [13], Eatock Taylor and Chau [14], Malenica, Eatock Taylor and Huang [15]. It is interesting to see what was observed in the work of Maniar and Newman [16]. They considered the wave diffraction by a large array of cylinders, and found that a very large wave force could be exerted on middle cylinders when the wavenumber was close to the trapped mode (e.g. Ursell [17]). Evans and Porter [18] further studied the wave force and mean second-order drift forces on vertical cylinders in circular arrangement and found large forces at certain frequencies. Duclos and Clément [19] considered cylinders randomly displaced from regular arrays based on a disorder parameter and found that a small level of spacing irregularity can greatly reduce the large forces on cylinders. Instead of regular incident waves, Walker and Eatock Taylor [20] investigated the diffraction of a NewWave, a focused wave group used to predict extreme situations from random wave spectra, from an array of cylinders, and large forces and free surface elevations were found to occur. While the above work is all conducted in the frequency domain, Wang and $\mathrm{Wu}$ [21] adopted a finite element method in the time domain to investigate the second-order water wave diffraction by an array or a group of vertical cylinders.

In addition to the work above on interaction between cylinders with the free surface waves, the interaction of hydroelastic waves with ocean structures also has become increasingly relevant and important for polar engineering. These kinds of problems elicited strong interest from many authors, for example: two-dimensional works by Sturova [22-24], Ren et al. [25], and Li et al. [26-28] — for a submerged/floating body in a polynya; and similar threedimensional work by Ren et al. [29]. In some cases, the free surface in the polynya may become frozen as well, and the surface of offshore structures becomes directly connected to the edge of ice sheet. The two-dimensional problem of hydroelastic waves interacting with vertical wall was investigated by Brocklehurst et al. [30] and Bhattacharjee and Soares [31]. For three-dimensional problems, Malenica et al. [32] investigated wave scattering of a bottommounted vertical cylinder frozen in a finite annular ice sheet by the eigenfunction expansion method, with the ice edge being clamped into the cylinder, while at the other end, the edge contacting open water is assumed free. Brocklehurst et al. [33] further considered a single vertical cylinder piercing through an ice sheet of infinite extent using a Weber transform. Results for the deflection and strain of the ice sheet, the horizontal wave forces as well as the vertical shear forces due to the clamped edge were provided. Recently, Korobkin et al. [34] further considered hydroelastic waves scattering by a vertical cylinder of an arbitrary cross section using a vertical mode method.

In the present work, we consider the diffraction of hydroelastic waves with multiple cylinders. The ice edge, or the intersection of the ice sheet with the cylinder surface, is not limited to clamped condition, and other edge conditions including free or simply supported can be incorporated into the formulation. Based on the method of eigenfunctions expansions and using the Green's second identity in an artificial domain, the problem can be solved in a highly effective manner. The paper is organized as below. In Sects. 2 and 3, we outline respectively the mathematical formulation and the solution procedure of the present problem. In Sect. 4, the results and discussions are provided for cylinders in different arrangements, including four cylinders in square arrangement, cylinders in one array and cylinders in two side by side arrays. Conclusions are provided in Sect. 5. 


\section{Mathematical formulation}

The coordinate systems used in the present work are introduced first. For each cylinder $j$, we set up a local cylindrical coordinate system $\left(r_{j}, \theta_{j}, z\right)$, with its origin on the mean free surface, and its $z$-axis along the centre line of the cylinder and pointing upwards. Apart from the local coordinate systems, fixed global Cartesian and cylindrical coordinate systems are also defined, respectively, as $O-x y z$ and $O-r \theta z$, with $x=r \cos \theta$ and $y=r \sin \theta$. The radius for cylinder $j$ is denoted as $c_{j}$. The centre of cylinder $j$ is at $\left(X_{j}, Y_{j}\right)$ in the global Cartesian coordinate system.

Based on the assumption that the fluid below the ice is inviscid and incompressible, and the flow is irrotational, the velocity potential theory can be adopted. For waves sinusoidal in time, the potential can be written as

$\Phi(x, y, z, t)=\operatorname{Re}\left\{\phi(x, y, z) \times \mathrm{e}^{-\mathrm{i} \omega t}\right\}$,

$\phi$ satisfies the governing equation

$\nabla^{2} \phi+\frac{\partial^{2} \phi}{\partial z^{2}}=0$

in the fluid domain, where $\nabla^{2}$ is the two-dimensional Laplacian defined as

$\nabla^{2}=\frac{\partial^{2}}{\partial x^{2}}+\frac{\partial^{2}}{\partial y^{2}}=\frac{\partial^{2}}{\partial r^{2}}+\frac{1}{r} \frac{\partial}{\partial r}+\frac{1}{r^{2}} \frac{\partial^{2}}{\partial \theta^{2}}$.

The linearized boundary condition on the ice sheet $S_{\text {ice }}$ can be written as Fox and Squire [35]

$\left(L \nabla^{4}-m_{\text {ice }} \omega^{2}+\rho g\right) \frac{\partial \phi}{\partial z}=\rho \omega^{2} \phi, \quad z=0, r_{j}>c_{j}$ for $j=1,2, \ldots, N C$,

where $L=E h^{3} /\left[12\left(1-v^{2}\right)\right]$ and $m_{\text {ice }}=\rho_{\text {ice }} h$ are, respectively, the effective flexural rigidity and the mass per unit area of the ice sheet. $E, v$ and $\rho_{\text {ice }}$ are the Young's modulus, Poisson's ratio and density of the ice, respectively. In the present work, these physical parameters have been taken as constants. $\rho$ in the equation is the density of water and $g$ is the acceleration due to gravity.

On the flat seabed $S_{B}(z=-H)$ and the wetted surfaces $S_{k 0}$ of the rigid cylinder $k\left(r_{k}=c_{k}, k=1,2, \ldots, N C\right)$, we have

$\frac{\partial \phi}{\partial n}=0$,

where $\boldsymbol{n}$ is the normal vector pointing out of the fluid domain. In the far-field, the radiation boundary condition on $S_{\infty}$ gives

$\frac{\partial \phi_{d}}{\partial r}=\mathrm{i} \kappa_{0} \phi_{d} \quad$ as $\quad r \rightarrow \infty$

to ensure that the disturbed wave will propagate outwards, where $\phi_{d}$ refers to the total diffraction potential below the ice sheet and $\kappa_{0}$ is the wavenumber. In addition to all these, edge conditions should also be satisfied at the intersection of the ice sheet with each cylinder, depending on whether the ice edge is clamped, free or simply supported. In present work, different edge conditions can be incorporated into the formulation, and case studies are made for the clamped edge and free edge conditions. For the former, there will be no deflection and slope on the ice edges, which can be given as (e.g. Timoshenko and Woinowsky-Krieger [36])

$\frac{\partial \phi}{\partial z}=0 \quad$ and $\quad \frac{\partial}{\partial r_{k}} \frac{\partial \phi}{\partial z}=0 \quad\left(r_{k}=c_{k}\right)$. 
For the latter, there is no bending moment and shear force on the ice edges. We have (e.g. Timoshenko and Woinowsky-Krieger [36])

$$
\begin{aligned}
& \left.\nabla^{2} \frac{\partial \phi}{\partial z}\right|_{z=0, r_{k}=c_{k}}=\left\{\frac{(1-v)}{c_{k}}\left\{\frac{1}{c_{k}} \frac{\partial^{2}}{\partial \theta_{k}^{2}}+\frac{\partial}{\partial r_{k}}\right\} \frac{\partial \phi}{\partial z}\right\}_{z=0, r_{k}=c_{k}}, \\
& \left.\frac{\partial}{\partial r_{k}}\left\{\nabla^{2} \frac{\partial \phi}{\partial z}\right\}\right|_{z=0, r_{k}=c_{k}}=\left\{-\frac{(1-v)}{c_{k}^{2}}\left(\frac{\partial^{3}}{\partial \theta_{k}^{2} \partial r_{k}}-\frac{1}{c_{k}} \frac{\partial^{2}}{\partial \theta_{k}^{2}}\right) \frac{\partial \phi}{\partial z}\right\}_{z=0, r_{k}=c_{k}} .
\end{aligned}
$$

\section{Solution procedure}

The incident potential due to a periodic wave from infinity can be written in the global system as

$\phi_{I}(x, y, z)=-A \frac{\mathrm{i} g}{\omega} \times \frac{\cosh \kappa_{0}(H+z)}{\cosh \kappa_{0} H} \times \mathrm{e}^{\mathrm{i} \kappa_{0}(x \cos \beta+y \sin \beta)}$,

where $A$ is the amplitude of the incident wave and $\beta$ is the angle of incidence relative to $x$ axis. In the local cylindrical system corresponding to cylinder $j$, this can be also written as

$\phi_{I}\left(r_{j}, \theta_{j}, z\right)=-A \frac{\mathrm{i} g}{\omega} \times \frac{\cosh \kappa_{0}(H+z)}{\cosh \kappa_{0} H} \times \mathrm{e}^{\mathrm{i} \kappa_{0} R_{j} \cos \left(\Theta_{j}-\beta\right)} \times \mathrm{e}^{\mathrm{i} \kappa_{0} r_{j} \cos \left(\theta_{j}-\beta\right)}$,

which can be expanded in a series of Bessel function as Abramowitz and Stegun [37]

$\phi_{I}\left(r_{j}, \theta_{j}, z\right)=-A \frac{\mathrm{i} g}{\omega} \times \frac{\cosh \kappa_{0}(H+z)}{\cosh \kappa_{0} H} \times \mathrm{e}^{\mathrm{i} \kappa_{0} R_{j} \cos \left(\Theta_{j}-\beta\right)} \times \sum_{m=-\infty}^{\infty} \mathrm{i}^{m} \times J_{m}\left(\kappa_{0} r_{j}\right) \times \mathrm{e}^{\mathrm{i} m\left(\theta_{j}-\beta\right)}$,

where $\left(R_{j}, \Theta_{j}\right)$ are the horizontal coordinates of the centre of cylinder $j$ in the global cylindrical system, with $X_{j}=R_{j} \cos \Theta_{j}, Y_{j}=R_{j} \sin \Theta_{j}$. The diffraction potential due to cylinder $j$ can be written through the Hankel functions $H_{m}^{(1)}$ as

$\phi_{D j}\left(r_{j}, \theta_{j}, z\right)=\sum_{n=-2}^{\infty} \sum_{m=-\infty}^{\infty} a_{n m}^{(j)} \times \psi_{n m}^{(j)}$

where

$\psi_{n m}^{(j)}=\frac{H_{m}^{(1)}\left(\kappa_{n} r_{j}\right)}{H_{m}^{(1)}\left(\kappa_{n} c_{j}\right)} \times \mathrm{e}^{\mathrm{i} m \theta_{j}} \times \frac{\cosh \kappa_{n}(z+H)}{\cosh \kappa_{n} H}$

and $\kappa_{n}$ are the solutions of the dispersion equation given below: (e.g. Fox and Squire [35])

$\kappa_{n} \times \tanh \kappa_{n} H=\frac{\rho \omega^{2}}{L \kappa_{n}^{4}+\rho g-m_{\mathrm{ice}} \omega^{2}}, \quad n=-2,-1,0,1,2 \ldots$

in which $\kappa_{0}$ is the positive real root, $\kappa_{-2}$ and $\kappa_{-1}$ are the two complex roots with positive imaginary part, and $\kappa_{n} \quad(n=1,2,3 \ldots)$ are the positive pure imaginary roots. Here $\psi_{n m}^{(j)}$ satisfies the boundary conditions on the bottom, ice sheet and at infinity. The unknown coefficients $a_{n m}^{(j)}$ in Eq. (11) are to be determined by the body surface and the edge boundary conditions.

The total diffraction potential below the ice sheet can be expressed as the summation of the individual diffracted potentials due to each cylinder relative to the local system $\left(r_{k}, \theta_{k}\right)$ as 


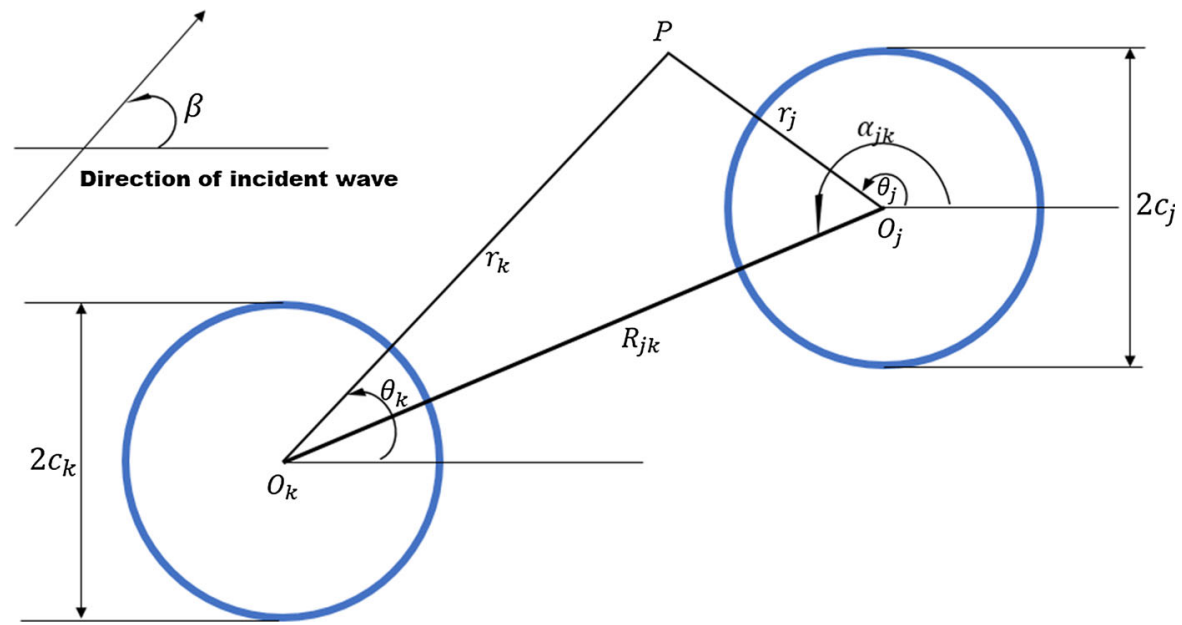

Fig. 1 The definition of parameters for Graf's addition theorem

$\phi_{d}(r, \theta, z)=\sum_{j=1}^{N C} \phi_{D j}\left(r_{j}, \theta_{j}, z\right)$.

To impose the body surface boundary condition on cylinder $k$, the potential in Eq. (14) should be written in terms $\left(r_{k}, \theta_{k}, z\right)$. To do that, the diffraction potential $\phi_{D j}$ in the cylindrical coordinate system $\left(r_{j}, \theta_{j}, z\right)$ should be transformed into the cylindrical system $\left(r_{k}, \theta_{k}, z\right)$. Here we define $\left(\alpha_{j k}, R_{j k}\right)$ as the polar coordinates of $O_{k}$ with respect to $O_{j}$. Particularly, $\alpha_{j k}$ is defined as an angle between the line linking $O_{j} O_{k}$ and $x$ axis, as shown in Fig. 1. They can be determined by $X_{k}-X_{j}=R_{j k} \cos \alpha_{j k}$ and $Y_{k}-Y_{j}=R_{j k} \sin \alpha_{j k}$.

Using the Graf's addition theorem for Bessel functions (e.g. Abramowitz and Stegun [37]), we have $H_{m}^{(1)}\left(\kappa_{n} r_{j}\right) \times \mathrm{e}^{\mathrm{i} m \theta_{j}}=\sum_{l=-\infty}^{\infty} H_{m+l}^{(1)}\left(\kappa_{n} R_{j k}\right) \times J_{l}\left(\kappa_{n} r_{k}\right) \times(-1)^{l} \times \mathrm{e}^{-\mathrm{i} l \theta_{k}} \times \mathrm{e}^{\mathrm{i}(m+l) \alpha_{j k}}, \quad j \neq k$.

It should be noted that as the above equation requires $R_{j k}>r_{k}$, it does not apply to the case of $j=k$. Then Eq. (14) can be transformed into the following form:

$\phi_{d}\left(r_{k}, \theta_{k}, z\right)=\sum_{n=-2}^{\infty} \sum_{m=-\infty}^{\infty}\left(a_{n m}^{(k)} \times \psi_{n m}^{(k)}+\sum_{j=1, j \neq k}^{N C} a_{n m}^{(j)} \times \xi_{n m}^{(j)}\right)$,

where

$\xi_{n m}^{(j)}=\frac{1}{H_{m}^{(1)}\left(\kappa_{n} c_{j}\right)} \times \frac{\cosh \kappa_{n}(z+H)}{\cosh \kappa_{n} H} \times \sum_{l=-\infty}^{\infty}\left(H_{m+l}^{(1)}\left(\kappa_{n} R_{j k}\right) \times J_{l}\left(\kappa_{n} r_{k}\right) \times(-1)^{l} \times \mathrm{e}^{-\mathrm{i} l \theta_{k}} \times \mathrm{e}^{\mathrm{i}(m+l) \alpha_{j k}}\right)$.

In the present work, we use the Green's second identity to impose boundary conditions on each cylinder surface and at the edge. For cylinder $k$, we have

$\oiint_{S}\left[\phi \frac{\partial \psi}{\partial n}-\psi \frac{\partial \phi}{\partial n}\right] \mathrm{d} S=0$,

where $\phi=\phi_{D k}, \psi=\psi_{n^{\prime},-m^{\prime}}^{(k)}$, and $S=S_{k 0}+S_{\infty}+S_{B}+S_{I}-S_{k F}$ is the entire boundary outside the cylinder $k$. $S_{k 0}$ is the wetted surface of the $k$ th cylinder as defined before Eq. (4). $S_{k F}$ is the cross section of cylinder $k$ cut 
though by the ice sheet, and $S_{I}$ is the entire upper surface of $z=0$. It is worth mentioning that we have included the ice surface occupied by all other cylinders in $S$, which makes the procedure different from the conventional one. The reason is that the Green's second identity can be valid for any closed boundary, if there is no singularity in $\varphi$ and $\psi$ on $S$ and in its closed domain. Based on the boundary conditions of the bottom surface $S_{B}$ and the far-field surface $S_{\infty}$, the integrals over these two surfaces are equal to zero. Also, as $\phi_{D k}$ and $\psi_{n^{\prime},-m^{\prime}}^{(k)}$ satisfy the boundary condition in Eq. (3) on $S_{I}-S_{k F}$, it allows the surface integral over $S_{I}-S_{k F}$ to be converted into a line integral of the edge of cylinder $k$ only based on the Gauss theorem. Following Ren et al. [29], Eq. (17) can then be written as

$$
\begin{aligned}
& -\iint_{S_{k}}\left[\phi_{D k} \frac{\partial \psi_{n^{\prime},-m^{\prime}}^{(k)}}{\partial r_{k}}-\psi_{n^{\prime},-m^{\prime}}^{(k)} \frac{\partial \phi_{D k}}{\partial r_{k}}\right] \mathrm{d} S \\
& -\frac{L}{\rho \omega^{2}} \times \oint_{c}\left(\begin{array}{l}
\frac{\partial \psi_{n^{\prime},-m^{\prime}}^{(k)}}{\partial z} \times \frac{\partial}{\partial r_{k}} \nabla^{2} \frac{\partial \phi_{D k}}{\partial z}-\frac{\partial}{\partial r_{k}} \frac{\partial \psi_{n^{\prime},-m^{\prime}}^{(k)}}{\partial z} \times \nabla^{2} \frac{\partial \phi_{D k}}{\partial z} \\
\left.-\frac{\partial \phi_{D k}}{\partial z} \times \frac{\partial}{\partial r_{k}} \nabla^{2} \frac{\partial \psi_{n^{\prime},-m^{\prime}}^{(k)}}{\partial z}+\frac{\partial}{\partial r_{k}} \frac{\partial \phi_{D k}}{\partial z} \times \nabla^{2} \frac{\partial \psi_{n^{\prime},-m^{\prime}}^{(k)}}{\partial z}\right)_{r_{k}=c_{k}, z=0} c_{k} \mathrm{~d} \theta_{k}=0 .
\end{array}\right.
\end{aligned}
$$

Noticing that $\phi_{D k}=\phi-\phi_{I}-\sum_{j=1, j \neq k}^{N C} \phi_{D j}$, where $\phi$ is the potential in Eq. (2) near cylinder $k$, and using the relationship obtained the Laplace equation $\nabla^{2} \phi=\left(\frac{\partial^{2}}{\partial r_{k}^{2}}+\frac{1}{r_{k}} \frac{\partial}{\partial r_{k}}+\frac{1}{r_{k}^{2}} \frac{\partial^{2}}{\partial \theta_{k}^{2}}\right) \phi=-\frac{\partial^{2}}{\partial z^{2}} \phi$, Eq. (18) can be further written as

$$
\begin{aligned}
& \int_{0}^{2 \pi}\left\{\begin{array}{l}
\int_{-H}^{0}\left(\frac{\partial \phi}{\partial r_{k}} \times \psi_{n^{\prime},-m^{\prime}}^{(k)}-\phi_{D k} \times \frac{\partial \psi_{n^{\prime},-m^{\prime}}^{(k)}}{\partial r_{k}}\right) \mathrm{d} z \\
-\frac{L}{\rho \omega^{2}} \times\left(\begin{array}{l}
-\frac{\partial \psi_{n^{\prime},-m^{\prime}}^{(k)}}{\partial z} \times \frac{\partial}{\partial r_{k}} \frac{\partial^{3} \phi}{\partial z^{3}}+\frac{\partial}{\partial r_{k}} \frac{\partial \psi_{n^{\prime},-m^{\prime}}^{(k)}}{\partial z} \times \frac{\partial^{3} \phi}{\partial z^{3}} \\
\left.+\frac{\partial \phi}{\partial z} \times \frac{\partial}{\partial r_{k}} \frac{\partial^{3} \psi_{n^{\prime},-m^{\prime}}^{(k)}}{\partial z^{3}}-\frac{\partial}{\partial r_{k}} \frac{\partial \phi}{\partial z} \times \frac{\partial^{3} \psi_{n^{\prime},-m^{\prime}}^{(k)}}{\partial z^{3}}\right)_{r_{k}=c_{k}, z=0}
\end{array}\right.
\end{array}\right\} \mathrm{d} \theta_{k} \\
& =\int_{0}^{2 \pi}\left\{\begin{array}{l}
\int_{-H}^{0}\left(\frac{\partial}{\partial r_{k}}\left(\phi_{I}+\sum_{j=1, j \neq k}^{N C} \phi_{D j}\right) \times \psi_{n^{\prime},-m^{\prime}}^{(k)}\right) \mathrm{d} z \\
-\frac{L}{\rho \omega^{2}} \times\left(\begin{array}{l}
-\frac{\partial \psi_{n^{\prime},-m^{\prime}}^{(k)}}{\partial z} \times \frac{\partial}{\partial r_{k}} \frac{\partial^{3}}{\partial z^{3}}\left(\phi_{I}+\sum_{j=1, j \neq k}^{N C} \phi_{D j}\right) \\
+\frac{\partial}{\partial r_{k}} \frac{\partial \psi_{n^{\prime},-m^{\prime}}^{(k)}}{\partial z} \times \frac{\partial^{3}}{\partial z^{3}}\left(\phi_{I}+\sum_{j=1, j \neq k}^{N C} \phi_{D j}\right) \\
+\frac{\partial}{\partial z}\left(\phi_{I}+\sum_{j=1, j \neq k}^{N C} \phi_{D j}\right) \times \frac{\partial}{\partial r_{k}} \frac{\partial^{3} \psi_{n^{\prime},-m^{\prime}}^{(k)}}{\partial z^{3}} \\
-\frac{\partial}{\partial r_{k}} \frac{\partial}{\partial z}\left(\phi_{I}+\sum_{j=1, j \neq k}^{N C} \phi_{D j}\right) \times \frac{\partial^{3} \psi_{n^{\prime},-m^{\prime}}^{(k)}}{\partial z^{3}}
\end{array}\right)_{r_{k}=c_{k}, z=0}
\end{array}\right\} \mathrm{d} \theta_{k}
\end{aligned}
$$

An advantage of using Green's second identity is that it makes application for the edge conditions easier. On the left-hand side of Eq. (19), the terms in the linear line integral involving various derivatives can be directly replaced by the corresponding edge conditions, see Eq. (6) or (7). The body surface boundary on the cylinder surface in Eq. (4) can be used in the first term in the surface integral. For the terms in the line integral, different edge conditions, depending on whether the edge is clamped, free or simply supported, can be incorporated. 
To treat with the clamped edge case, we substitute the body surface condition and clamped edge conditions (Eq. 6) into Eq. (19); by taking all the terms of $\frac{\partial \phi}{\partial r_{k}}, \frac{\partial \phi}{\partial z}$ and $\frac{\partial}{\partial r_{k}} \frac{\partial \phi}{\partial z}$ as zero, we can obtain

$$
\begin{aligned}
& \int_{0}^{2 \pi}\left\{\int_{-H}^{0}\left(-\phi_{D k} \times \frac{\partial \psi_{n^{\prime},-m^{\prime}}^{(k)}}{\partial r_{k}}\right) \mathrm{d} z-\frac{L}{\rho \omega^{2}} \times\left(-\frac{\partial \psi_{n^{\prime},-m^{\prime}}^{(k)}}{\partial z} \times \frac{\partial}{\partial r_{k}} \frac{\partial^{3} \phi_{D k}}{\partial z^{3}}+\frac{\partial}{\partial r_{k}} \frac{\partial \psi_{n^{\prime},-m^{\prime}}^{(k)}}{\partial z} \times \frac{\partial^{3} \phi_{D k}}{\partial z^{3}}\right)_{r_{k}=c_{k}, z=0}\right\} \mathrm{d} \theta_{k} \\
& =\int_{0}^{2 \pi}\left\{\begin{array}{l}
\int_{-H}^{0}\left(\frac{\partial}{\partial r_{k}}\left(\phi_{I}+\sum_{j=1, j \neq k}^{N C} \phi_{D j}\right) \times \psi_{n^{\prime},-m^{\prime}}^{(k)}\right) \mathrm{d} z \\
-\frac{L}{\rho \omega^{2}} \times\left(\frac{\partial}{\partial z}\left(\phi_{I}+\sum_{j=1, j \neq k}^{N C} \phi_{D j}\right) \times \frac{\partial}{\partial r_{k}} \frac{\partial^{3} \psi_{n^{\prime},-m^{\prime}}^{(k)}}{\partial z^{3}}\right. \\
\left.-\frac{\partial}{\partial r_{k}} \frac{\partial}{\partial z}\left(\phi_{I}+\sum_{j=1, j \neq k}^{N C} \phi_{D j}\right) \times \frac{\partial^{3} \psi_{n^{\prime},-m^{\prime}}^{(k)}}{\partial z^{3}}\right)_{r_{k}=c_{k}, z=0}
\end{array}\right\} \mathrm{d} \theta_{k} .
\end{aligned}
$$

Finally, by substituting Eqs. (10)-(16) into Eq. (20) and performing the integrations with respect to $\theta_{k}$ and $z$, we can obtain a system of linear equations as below:

$$
\begin{aligned}
& \sum_{n=-2}^{\infty} a_{n m^{\prime}}^{(k)} \times A^{(k)}\left(n, n^{\prime}, m^{\prime}\right) \\
& \quad+\sum_{j=1, j \neq k}^{N C} \sum_{n=-2}^{\infty} \sum_{m=-\infty}^{\infty} a_{n m}^{(j)} \times \frac{H_{m-m^{\prime}}^{(1)}\left(\kappa_{n} R_{j k}\right) \times(-1)^{-m^{\prime}} \times \mathrm{e}^{\mathrm{i}\left(m-m^{\prime}\right) \alpha_{j k}}}{H_{m}^{(1)}\left(\kappa_{n} c_{j}\right)} \times B^{(k)}\left(n, n^{\prime}, m^{\prime}\right) \\
& =A \frac{\mathrm{i} g}{\omega} \times \mathrm{e}^{\mathrm{i} \kappa_{0} R_{k} \cos \left(\Theta_{k}-\beta\right)} \times \mathrm{i}^{m^{\prime}} \times \mathrm{e}^{-\mathrm{i} m^{\prime} \beta} \times C^{(k)}\left(n, n^{\prime}, m^{\prime}\right),
\end{aligned}
$$

where

$$
\begin{aligned}
A^{(k)}\left(n, n^{\prime}, m^{\prime}\right)= & \kappa_{n^{\prime}} \times \frac{H_{-m^{\prime}}^{(1)^{\prime}}\left(\kappa_{n^{\prime}} c_{k}\right)}{H_{-m^{\prime}}^{(1)}\left(\kappa_{n^{\prime}} c_{k}\right)} \times X\left(\kappa_{n}, \kappa_{n^{\prime}}\right)+Y\left(\kappa_{n^{\prime}}, \kappa_{n}\right) \\
& \times\left(\kappa_{n^{\prime}} \times \frac{H_{-m^{\prime}}^{(1)^{\prime}}\left(\kappa_{n^{\prime}} c_{k}\right)}{H_{-m^{\prime}}^{(1)}\left(\kappa_{n^{\prime}} c_{k}\right)}-\kappa_{n} \times \frac{H_{m^{\prime}}^{(1)^{\prime}}\left(\kappa_{n} c_{k}\right)}{H_{m^{\prime}}^{(1)}\left(\kappa_{n} c_{k}\right)}\right), \\
B^{(k)}\left(n, n^{\prime}, m^{\prime}\right)= & X\left(\kappa_{n}, \kappa_{n^{\prime}}\right) \times \kappa_{n} \times J_{-m^{\prime}}^{\prime}\left(\kappa_{n} c_{k}\right)-Y\left(\kappa_{n}, \kappa_{n^{\prime}}\right) \\
& \times\left(J_{-m^{\prime}}\left(\kappa_{n} c_{k}\right) \times \kappa_{n^{\prime}} \times \frac{H_{-m^{\prime}}^{(1)^{\prime}}\left(\kappa_{n^{\prime}} c_{k}\right)}{H_{-m^{\prime}}^{(1)}\left(\kappa_{n^{\prime}} c_{k}\right)}-\kappa_{n} \times J_{-m^{\prime}}^{\prime}\left(\kappa_{n} c_{k}\right)\right), \\
C^{(k)}\left(n, n^{\prime}, m^{\prime}\right)= & X\left(\kappa_{0}, \kappa_{n^{\prime}}\right) \times \kappa_{0} \times J_{m^{\prime}}^{\prime}\left(\kappa_{0} c_{k}\right)+Y\left(\kappa_{0}, \kappa_{n^{\prime}}\right) \\
& \times\left(\kappa_{0} \times J_{m^{\prime}}^{\prime}\left(\kappa_{0} c_{k}\right)-J_{m^{\prime}}\left(\kappa_{0} c_{k}\right) \times \kappa_{n^{\prime}} \times \frac{H_{-m^{\prime}}^{(1)^{\prime}}\left(\kappa_{n^{\prime}} c_{k}\right)}{H_{-m^{\prime}}^{(1)}\left(\kappa_{n^{\prime}} c_{k}\right)}\right)
\end{aligned}
$$

and

$X\left(x_{1}, x_{2}\right)=\frac{x_{1} \tanh x_{1} H-x_{2} \tanh x_{2} H}{x_{1}^{2}-x_{2}^{2}}, \quad Y\left(x_{1}, x_{2}\right)=\frac{L}{\rho \omega^{2}} \times x_{1} \times \tanh x_{1} H \times x_{2}^{3} \times \tanh x_{2} H$.

We may notice that Eq. (21) is non-diagonal. This means that integral in the vertical direction based on the Green second identity is non-orthogonal. There have been some attempts to use orthogonal inner product (Sahoo et al. [38]). However, the final matrix is not diagonal in this case. In fact, it is common for an orthogonal inner product, 
when the potential or the derivative of the potential is entirely replaced by the boundary conditions to form an equation, the left-hand side of the identity will have only one unknown, while the right-hand side is known. As a result, the final matrix equation will be diagonal. In this case, however, the potential or the derivative in the surface integral is replaced by another unknown potential or derivative based on the continuity condition. The right-hand side therefore involves also the unknowns. Thus, the overall matrix is not diagonal.

For the free-edge case, the free edge conditions can be applied through replacing the terms $\frac{\partial^{3}}{\partial z^{3}} \frac{\partial \phi}{\partial r_{k}}$ and $\frac{\partial^{3} \phi}{\partial z^{3}}$ on the left side of equal sign respectively by $-\frac{\partial}{\partial r_{k}}\left\{\nabla^{2} \frac{\partial \phi}{\partial z}\right\}$ and $-\nabla^{2} \frac{\partial \phi}{\partial z}$ in Eq. (19). When we substitute the body surface condition and free edge conditions into Eq. (19), we can obtain

$$
\begin{aligned}
& \int_{0}^{2 \pi}\left\{\int_{-H}^{0}\left(-\phi_{D k} \times \frac{\partial \psi_{n^{\prime},-m^{\prime}}^{(k)}}{\partial r_{k}}\right) \mathrm{d} z-\frac{L}{\rho \omega^{2}} \times\left(\begin{array}{l}
-\frac{\partial \psi_{n^{\prime},-m^{\prime}}^{(k)}}{\partial z} \times \frac{(1-v)}{c_{k}^{2}}\left(\frac{\partial^{3}}{\partial \theta_{k}^{2} \partial r_{k}}-\frac{1}{c_{k}} \frac{\partial^{2}}{\partial \theta_{k}^{2}}\right) \frac{\partial \phi}{\partial z} \\
-\frac{\partial}{\partial r_{k}} \frac{\partial \psi_{n^{\prime},-m^{\prime}}^{(k)}}{\partial z} \times \frac{(1-v)}{c_{k}}\left\{\frac{1}{c_{k}} \frac{\partial^{2}}{\partial \theta_{k}^{2}}+\frac{\partial}{\partial r_{k}}\right\} \frac{\partial \phi}{\partial z} \\
\left.+\frac{\partial \phi_{D k}}{\partial z} \times \frac{\partial}{\partial r_{k}} \frac{\partial^{3} \psi_{n^{\prime},-m^{\prime}}^{(k)}}{\partial z^{3}}-\frac{\partial}{\partial r_{k}} \frac{\partial \phi_{D k}}{\partial z} \times \frac{\partial^{3} \psi_{n^{\prime},-m^{\prime}}^{(k)}}{\partial z^{3}}\right)
\end{array}\right.\right. \\
& =\int_{0}^{2 \pi}\left\{\begin{array}{l}
\int_{-H}^{0}\left(\frac{\partial}{\partial r_{k}}\left(\phi_{I}+\sum_{j=1, j \neq k}^{N C} \phi_{D j}\right) \times \psi_{n^{\prime},-m^{\prime}}^{(k)}\right) \mathrm{d} z \\
-\frac{L}{\rho \omega^{2}} \times\left(-\frac{\partial \psi_{n^{\prime},-m^{\prime}}^{(k)}}{\partial z} \times \frac{\partial}{\partial r_{k}} \frac{\partial^{3}}{\partial z^{3}}\left(\phi_{I}+\sum_{j=1, j \neq k}^{N C} \phi_{D j}\right)\right. \\
\left.+\frac{\partial}{\partial r_{k}} \frac{\partial \psi_{n^{\prime},-m^{\prime}}^{(k)}}{\partial z} \times \frac{\partial^{3}}{\partial z^{3}}\left(\phi_{I}+\sum_{j=1, j \neq k}^{N C} \phi_{D j}\right)\right)
\end{array}\right\} \mathrm{d} \theta .
\end{aligned}
$$

Similarly, by substituting Eqs. (10)-(16) into Eq. (22), we can obtain the system of linear equations as

$$
\begin{aligned}
& \sum_{n=-2}^{\infty} a_{n m^{\prime}}^{(k)} \times D^{(k)}\left(n, n^{\prime}, m^{\prime}\right)+\sum_{j=1, j \neq k}^{N C} \sum_{n=-2}^{\infty} \sum_{m=-\infty}^{\infty} a_{n m}^{(j)} \times \frac{1}{H_{m}^{(1)}\left(\kappa_{n} c_{j}\right)} \times H_{m-m^{\prime}}^{(1)}\left(\kappa_{n} R_{j k}\right) \times(-1)^{-m^{\prime}} \\
& \quad \times \mathrm{e}^{\mathrm{i}\left(m-m^{\prime}\right) \alpha_{j k}} \times E^{(k)}\left(n, n^{\prime}, m^{\prime}\right) \\
& =A \frac{\mathrm{i} g}{\omega} \times \mathrm{e}^{\mathrm{i} \kappa_{0} R_{k} \cos \left(\Theta_{k}-\beta\right)} \times \mathrm{i}^{m^{\prime}} \times \mathrm{e}^{-\mathrm{i} m^{\prime} \beta} \times F^{(k)}\left(n, n^{\prime}, m^{\prime}\right)
\end{aligned}
$$

where

$$
\begin{aligned}
D^{(k)}\left(n, n^{\prime}, m^{\prime}\right)= & \kappa_{n^{\prime}} \times \frac{H_{-m^{\prime}}^{(1)^{\prime}}\left(\kappa_{n^{\prime}} c_{k}\right)}{H_{-m^{\prime}}^{(1)}\left(\kappa_{n^{\prime}} c_{k}\right)} \times X\left(\kappa_{n}, \kappa_{n^{\prime}}\right)+Z\left(\kappa_{n}, \kappa_{n^{\prime}}\right) \\
& \times\left(\begin{array}{l}
\left(\kappa_{n} \times \frac{H_{m^{\prime}}^{(1)^{\prime}}\left(\kappa_{n} c_{k}\right)}{H_{m^{\prime}}^{(1)}\left(\kappa_{n} c_{k}\right)}-\frac{1}{c_{k}}\right) \times \frac{(1-v)}{c_{k}^{2}} \times m^{\prime 2} \\
+\left(\frac{m^{\prime 2}}{c_{k}}-\kappa_{n} \times \frac{H_{m^{\prime}}^{(1)^{\prime}}\left(\kappa_{n} c_{k}\right)}{H_{m^{\prime}}^{(1)}\left(\kappa_{n} c_{k}\right)}\right) \times \kappa_{n^{\prime}} \times \frac{H_{-m^{\prime}}^{(1)^{\prime}}\left(\kappa_{n^{\prime}} c_{k}\right)}{H_{-m^{\prime}}^{(1)}\left(\kappa_{n^{\prime}} c_{k}\right)} \times \frac{(1-v)}{c_{k}} \\
+\left(\kappa_{n^{\prime}} \times \frac{H_{-m^{\prime}}^{(1)^{\prime}}\left(\kappa_{n^{\prime}} c_{k}\right)}{H_{-m^{\prime}}^{(1)}\left(\kappa_{n^{\prime}} c_{k}\right)}-\kappa_{n} \times \frac{H_{m^{\prime}}^{(1)^{\prime}}\left(\kappa_{n} c_{k}\right)}{H_{m^{\prime}}^{(1)}\left(\kappa_{n} c_{k}\right)}\right) \times \kappa_{n^{\prime}}^{2}
\end{array}\right),
\end{aligned}
$$




$$
\begin{aligned}
& E^{(k)}\left(n, n^{\prime}, m^{\prime}\right)=\kappa_{n} \times J_{-m^{\prime}}^{\prime}\left(\kappa_{n} c_{k}\right) \times X\left(\kappa_{n}, \kappa_{n^{\prime}}\right)+Z\left(\kappa_{n}, \kappa_{n^{\prime}}\right) \\
& \times\left(\begin{array}{l}
\left(\begin{array}{l}
\kappa_{n} \times J_{-m^{\prime}}^{\prime}\left(\kappa_{n} c_{k}\right) \\
-J_{-m^{\prime}}\left(\kappa_{n} c_{k}\right) \times \kappa_{n^{\prime}} \times \frac{H_{-m^{\prime}}^{(1)^{\prime}}\left(\kappa_{n^{\prime}} c_{k}\right)}{H_{-m^{\prime}}^{(1)}\left(\kappa_{n^{\prime}} c_{k}\right)}
\end{array}\right) \times \kappa_{n}^{2} \\
+m^{\prime 2} \times\left(\begin{array}{l}
\kappa_{n} \times J_{-m^{\prime}}^{\prime}\left(\kappa_{n} c_{k}\right) \\
-\frac{1}{c_{k}} \times J_{-m^{\prime}}\left(\kappa_{n} c_{k}\right)
\end{array}\right) \times \frac{(1-v)}{c_{k}^{2}} \\
+\left(\begin{array}{l}
\frac{m^{\prime 2}}{c_{k}} \times J_{-m^{\prime}}\left(\kappa_{n} c_{k}\right) \\
-\kappa_{n} \times J_{-m^{\prime}}^{\prime}\left(\kappa_{n} c_{k}\right)
\end{array}\right) \times \kappa_{n^{\prime}} \times \frac{H_{-m^{\prime}}^{(1)^{\prime}}\left(\kappa_{n^{\prime}} c_{k}\right)}{H_{-m^{\prime}}^{(1)}\left(\kappa_{n^{\prime}} c_{k}\right)} \times \frac{(1-v)}{c_{k}}
\end{array}\right), \\
& F^{(k)}\left(n, n^{\prime}, m^{\prime}\right)=\kappa_{0} \times J_{m^{\prime}}^{\prime}\left(\kappa_{0} c_{k}\right) \times X\left(\kappa_{0}, \kappa_{n^{\prime}}\right)+Z\left(\kappa_{0}, \kappa_{n^{\prime}}\right) \\
& \times\left(\begin{array}{l}
\left(\begin{array}{l}
\kappa_{0} \times J_{m^{\prime}}^{\prime}\left(\kappa_{0} c_{k}\right) \\
-J_{m^{\prime}}\left(\kappa_{0} c_{k}\right) \times \kappa_{n^{\prime}} \times \frac{H_{-m^{\prime}}^{(1)^{\prime}}\left(\kappa_{n^{\prime}} c_{k}\right)}{H_{-m^{\prime}}^{(1)}\left(\kappa_{n^{\prime}} c_{k}\right)}
\end{array}\right) \times \kappa_{0}^{2} \\
+m^{\prime 2} \times\left(\begin{array}{l}
\kappa_{0} \times J_{m^{\prime}}^{\prime}\left(\kappa_{0} c_{k}\right) \\
-\frac{1}{c_{k}} \times J_{m^{\prime}}\left(\kappa_{0} c_{k}\right)
\end{array}\right) \times \frac{(1-v)}{c_{k}^{2}} \\
-\left(\begin{array}{l}
J_{m^{\prime}}\left(\kappa_{0} c_{k}\right) \times\left(-m^{\prime 2}\right) \times \frac{1}{c_{k}} \\
+\kappa_{0} \times J_{m^{\prime}}^{\prime}\left(\kappa_{0} c_{k}\right)
\end{array}\right) \times \kappa_{n^{\prime}} \times \frac{H_{-m^{\prime}}^{(1)^{\prime}}\left(\kappa_{n^{\prime}} c_{k}\right)}{H_{-m^{\prime}}^{(1)}\left(\kappa_{n^{\prime}} c_{k}\right)} \times \frac{(1-v)}{c_{k}}
\end{array}\right),
\end{aligned}
$$

and $Z\left(x_{1}, x_{2}\right)=\frac{L}{\rho \omega^{2}} \times x_{1} \times \tanh x_{1} H \times x_{2} \times \tanh x_{2} H$.

Through solving the system of linear equations given by Eqs. (21) and (23) respectively, we can obtain the unknown coefficients $a_{n m}^{(k)}, k=1,2, \ldots, N C$ for the clamped and free edge problems. In the practical calculation, the infinite series in Eq. (15) needs to be truncated. Specifically, $n$ ranges from -2 to $N-3$ for $\kappa_{n}$, and $m$ ranges from $-M$ to $M . N$ and $M$ should be chosen large enough to ensure that the convergence of the results has been achieved.

Once the velocity potential been obtained, the wave forces, or the horizontal forces, on cylinder $k$ can be calculated based on the following equation:

$$
\begin{aligned}
& \left\{\begin{array}{l}
F_{x}^{(k)} \\
F_{y}^{(k)}
\end{array}\right\}=\mathrm{i} \omega \rho \iint_{S_{k 0}} \phi \times\left\{\begin{array}{l}
n_{x} \\
n_{y}
\end{array}\right\} \mathrm{d} S \\
& =\left\{\begin{array}{l}
i \\
1
\end{array}\right\} \times \pi \omega \rho c_{k} \times\left\{\begin{array}{l}
A \frac{\mathrm{i} g}{\omega} \times \frac{\tanh \kappa_{0} H}{\kappa_{0}} \times \mathrm{e}^{\mathrm{i} \kappa_{0} R_{k} \cos \left(\Theta_{k}-\beta\right)} \times \mathrm{i} \\
\times\left(J_{-1}\left(\kappa_{0} c_{k}\right) \times \mathrm{e}^{\mathrm{i} \beta}+\left\{\begin{array}{c}
-1 \\
1
\end{array}\right\} \times J_{1}\left(\kappa_{0} c_{k}\right) \times \mathrm{e}^{-\mathrm{i} \beta}\right) \\
+\sum_{n=-2}^{\infty}\left(a_{n,-1}^{(k)}+\left\{\begin{array}{c}
1 \\
-1
\end{array}\right\} \times a_{n, 1}^{(k)}\right) \times \frac{\tanh \kappa_{n} H}{\kappa_{n}} \\
-\sum_{j=1, j \neq k}^{N C} \sum_{n=-2}^{\infty} \sum_{m=-\infty}^{\infty} \frac{a_{n m}^{(j)}}{H_{m}^{(1)}\left(\kappa_{n} c_{j}\right)} \times \frac{\tanh \kappa_{n} H}{\kappa_{n}} \\
\times\left(\begin{array}{c}
H_{m+1}^{(1)}\left(\kappa_{n} R_{j k}\right) \times J_{1}\left(\kappa_{n} c_{k}\right) \times \mathrm{e}^{\mathrm{i}(m+1) \alpha_{j k}} \\
+\left\{\begin{array}{c}
1 \\
-1
\end{array}\right\} \times H_{m-1}^{(1)}\left(\kappa_{n} R_{j k}\right) \times J_{-1}\left(\kappa_{n} c_{k}\right) \times \mathrm{e}^{\mathrm{i}(m-1) \alpha_{j k}}
\end{array}\right.
\end{array}\right\},
\end{aligned}
$$

where $n_{x}, n_{y}$ are, respectively, the components of the normal vector of the body surface pointing outwards, along $x$-axis and $y$-axis.

The vertical shear stress $Q_{k}$ can be given as (e.g. Eq. 2.2 in Ugural [39])

$Q_{k}=-L \times \frac{\partial}{\partial r_{k}}\left(\nabla^{2} w\right)_{r_{k}=c_{k}}$. 
Fig. 2 Force on middle body of an array of nine cylinders

$(\beta=0, c=H=1$,

$d=2, h=0, X_{j}=2$

$(j-1) d, Y_{j}=0, j=1$,

$2, \ldots 9)$

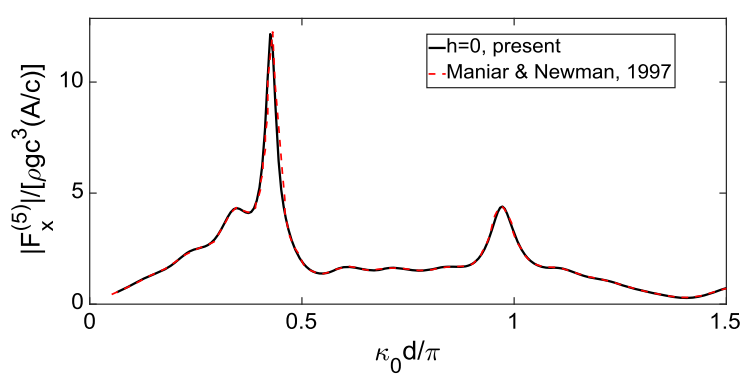

Substituting $w=\frac{\mathrm{i}}{\omega} \phi_{z}$ into (25), we have

$$
\begin{aligned}
& Q_{k}=-L \times \frac{\partial}{\partial r_{k}}\left(\nabla^{2} w\right)_{r_{k}=c_{k}} \\
&=L \times \frac{\mathrm{i}}{\omega} \times\left(\begin{array}{l}
-A \frac{\mathrm{i} g}{\omega} \times \kappa_{0}^{4} \times \tanh \kappa_{0} H \times \mathrm{e}^{\mathrm{i} \kappa_{0} R_{k} \cos \left(\Theta_{k}-\beta\right)} \times \sum_{m=-\infty}^{\infty} \mathrm{i}^{m} \times J_{m}^{\prime}\left(\kappa_{0} c_{k}\right) \times \mathrm{e}^{\mathrm{i} m\left(\theta_{k}-\beta\right)} \\
+\sum_{n=-2}^{\infty} \sum_{m=-\infty}^{\infty} a_{n m}^{(k)} \times \frac{H_{m}^{(1)^{\prime}}\left(\kappa_{n} c_{k}\right)}{H_{m}^{(1)}\left(\kappa_{n} c_{k}\right)} \times \mathrm{e}^{\mathrm{i} m \theta_{k}} \times \kappa_{n}^{4} \times \tanh \kappa_{n} H \\
+\sum_{n=-2}^{\infty} \sum_{m=-\infty}^{\infty} \sum_{j=1, j \neq k}^{N C} a_{n m}^{(j)} \times \frac{1}{H_{m}^{(1)}\left(\kappa_{n} c_{j}\right)} \times \kappa_{n}^{4} \times \tanh \kappa_{n} H \\
\times \sum_{l=-\infty}^{\infty} H_{m+l}^{(1)}\left(\kappa_{n} R_{j k}\right) \times J_{l}^{\prime}\left(\kappa_{n} c_{k}\right) \times(-1)^{l} \times \mathrm{e}^{-\mathrm{i} l \theta_{k}} \times \mathrm{e}^{\mathrm{i}(m+l) \alpha_{j k}}
\end{array}\right) .
\end{aligned}
$$

The shear force $Q_{t o t}^{(k)}$ on the cylinder can be obtained by integrating $Q_{k}$ along the intersection line of the cylinder with the ice sheet:

$$
\begin{aligned}
& Q_{\mathrm{tot}}^{(k)}= \int_{0}^{2 \pi} Q_{k} \times c_{k} \mathrm{~d} \theta_{k} \\
&=L \times \frac{\mathrm{i}}{\omega} \times 2 \pi c_{k} \times\left(\begin{array}{l}
-A \frac{\mathrm{i} g}{\omega} \times \kappa_{0}^{4} \times \tanh \kappa_{0} H \times \mathrm{e}^{\mathrm{i} \kappa_{0} R_{k} \cos \left(\Theta_{k}-\beta\right)} \times J_{0}^{\prime}\left(\kappa_{0} c_{k}\right) \\
+\sum_{n=-2}^{\infty} a_{n 0}^{(k)} \times \frac{H_{0}^{(1)^{\prime}}\left(\kappa_{n} c_{k}\right)}{H_{0}^{(1)}\left(\kappa_{n} c_{k}\right)} \times \kappa_{n}^{4} \times \tanh \kappa_{n} H \\
+\sum_{n=-2}^{\infty} \sum_{m=-\infty}^{\infty} \sum_{j=1, j \neq k}^{N C} a_{n m}^{(j)} \times \frac{1}{H_{m}^{(1)}\left(\kappa_{n} c_{j}\right)} \times \kappa_{n}^{4} \\
\times \tanh \kappa_{n} H \\
\times H_{m}^{(1)}\left(\kappa_{n} R_{j k}\right) \times J_{0}^{\prime}\left(\kappa_{n} c_{k}\right) \times \mathrm{e}^{\mathrm{i} m \alpha_{j k}}
\end{array}\right) .
\end{aligned}
$$

\section{Results and discussion}

\subsection{Validation}

We first consider a special case of $h=0$. In such a case, $n=-1,-2$ should be removed from Eq. (11). This is in fact the case of open water. Simulation is made for an array of 9 equally spaced cylinders of the same radius $c$ along the $x$-axis, with $X_{j}=2(j-1) d, Y_{j}=0, j=1,2, \ldots 9$. The wave force $F_{x}$ for $\beta=0$ and $d=2$ on the cylinder at the centre, or $j=5$, is provided in Fig. 2. Convergence has been achieved for $\mathrm{N}=10$ and $\mathrm{M}=10$. It can be found that our result is in good agreement with that of Maniar and Newman [16], including the local peaks physics of which have been discussed by Maniar and Newman [16].

We then consider a case of single cylinder with the radius of $c$ piercing through the ice sheet with the clamped edge. The problem has been solved by Brocklehurst et al. [33] using the Weber transform. We adopt the same parameters as those in Fig. 8 in [33]. To be consistent with the equation of incident wave potential in [33], we set $A$ in Eq. (6) as $\frac{\mathrm{i} a \omega^{2}}{g \kappa_{0} \times \tanh \kappa_{0} H}$ and $a=0.02 \mathrm{~m}$. The force $F_{x}$ from $N=20$, which has been found to provide converged results, against the wavenumber is provided in Fig. $3 \mathrm{a}$ and the comparison with that from [33] shows a 
Fig. 3 Forces on a single cylinder piercing through ice sheet with the clamped edge $(H=350 \mathrm{~m}$, $v=0.33$,

$\rho_{\text {ice }}=917 \mathrm{~kg} \mathrm{~m}^{-3}$

$\rho=1026 \mathrm{~kg} \mathrm{~m}^{-3}$,

$E=4.2 \times 10^{9} \mathrm{~N} \mathrm{~m}^{-2}$ ).

a Horizontal wave force

$(h=1.6 \mathrm{~m})$, b vertical shear

force $(c=3.5 \mathrm{~m})$
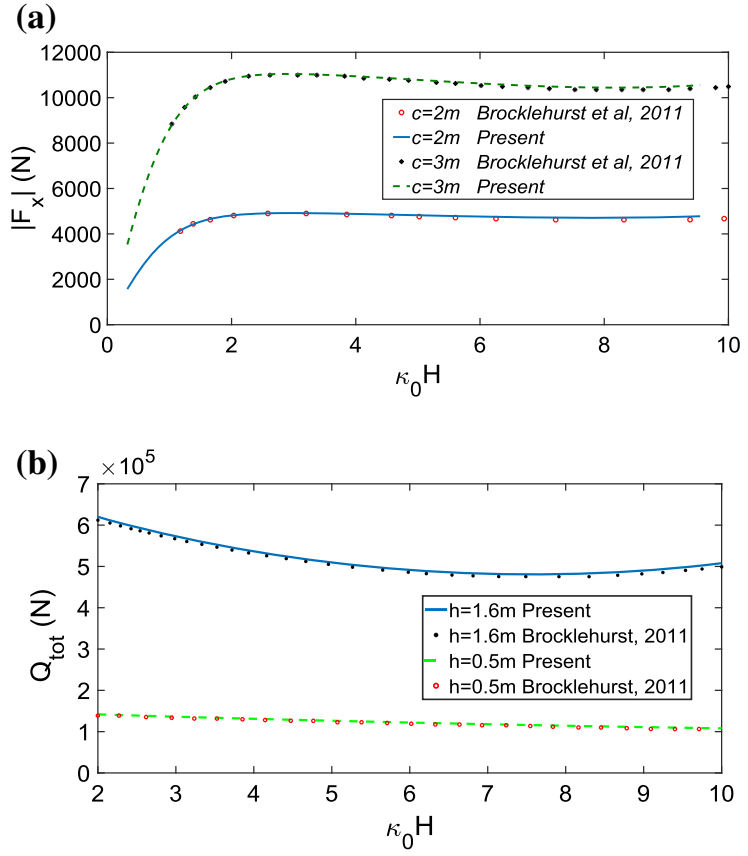

very good agreement. Comparison for the vertical shear force on a single cylinder is also made with that in Fig. 7 of Brocklehurst et al. [33] and shown in Fig. 3b. It can be seen that a good agreement has been achieved, which further verifies the present method.

\subsection{Four cylinders in square arrangement}

The typical values for parameters used in the present work are given below:

$E=5 \mathrm{GPa}, \quad v=0.3, \quad \rho_{\text {ice }}=922.5 \mathrm{~kg} \mathrm{~m}^{-3}, \quad \rho=1025 \mathrm{~kg} \mathrm{~m}^{-3}, \quad g=9.8 \mathrm{~m} / \mathrm{s}^{2}$

unless stated otherwise, which are consistent with those used in Sturova [24] and Ren et al. [25]. The nondimensionalization for the variables in our computation is based on the following basic parameters, namely, the gravitational acceleration $g$, the density of water $\rho$ as well as the radius of cylinder $c$. Therefore, the wave force is, for example, nondimensionalized by $\rho g c^{3}$, which is further normalized by $A / c$ as in Fig. 2.

For the first case study, we consider a configuration of four cylinders in square arrangement which are commonly seen on an offshore platform. The sketch of the configuration is given in Fig. 4a, b. The cylinders marked 1-4 are located at $(-\sqrt{2} d, 0),(0, \sqrt{2} d),(\sqrt{2} d, 0)$ and $(0,-\sqrt{2} d)$, respectively. We first consider the following sea, or $\beta=0$, which means that the wave comes from $x=-\infty$ and propagates along the positive direction of the $x$-axis.

In the present case, the geometric parameters are chosen as $h=0.1, d=2, H=10$ and $c_{j}=c, j=1,2,3,4$ where $c$ is used as the characteristic length. The wave forces on cylinders $1,2,3$ in the $x$ direction are shown in Fig. 5a-c, respectively, as the force on cylinder 4 is the same as that on cylinder 2 . Figure $5 \mathrm{~d}$ provides the force in the $y$ direction on cylinder 4, magnitude of which is the same as that on cylinder 2, while it is zero on cylinders 1 and 3. Results for both free and clamped edges are provided, together with those for the open water case for comparison.

At a given $\kappa_{0}, \omega$ is obtained from Eq. (13) at $n=0$. We can see that as $\omega$ is dependent on the ice thickness $h$, the incident potential in Eq. (9) is also dependent on $h$. However, in Eq. (25), we can see that its first term, corresponding to the contribution of incident potential to the force on the cylinder, is independent of $\omega$. This means that the contributions of the incident potential to the force in the open water and in the ice sheet are the same. At 
(a)

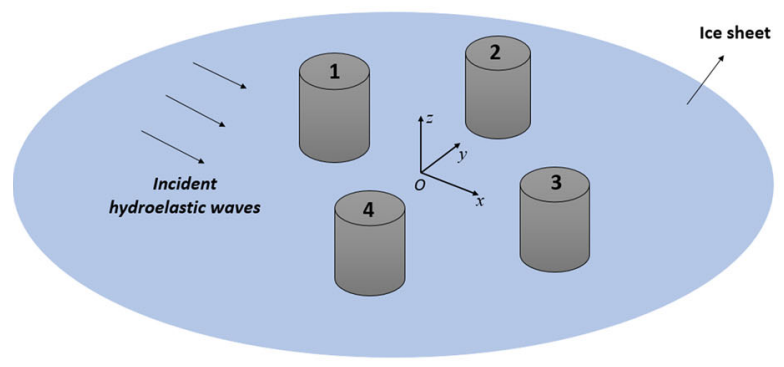

Fig. 4 Sketch of the four cylinders in incident hydroelastic waves
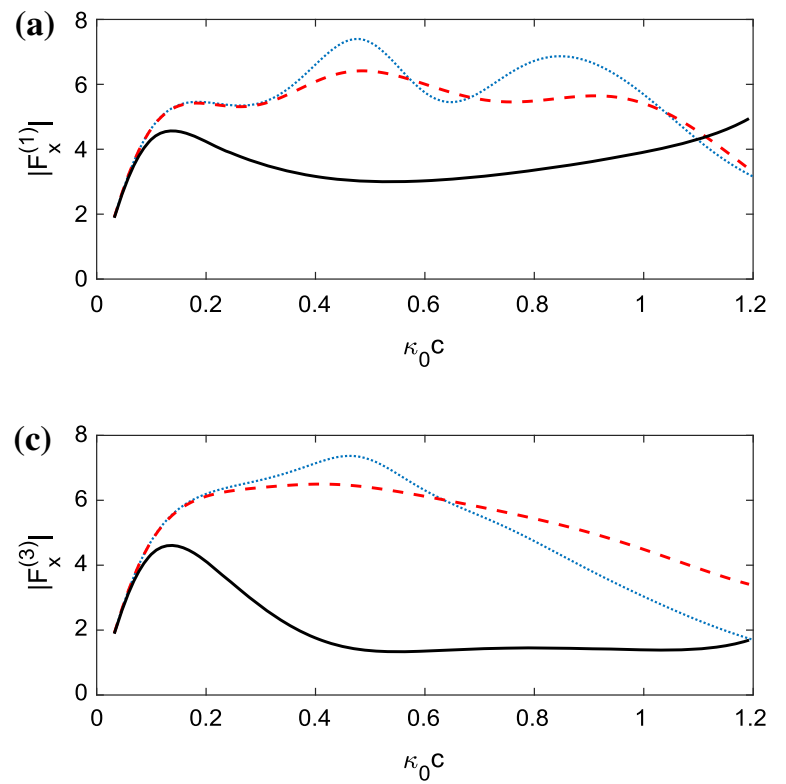

(b)
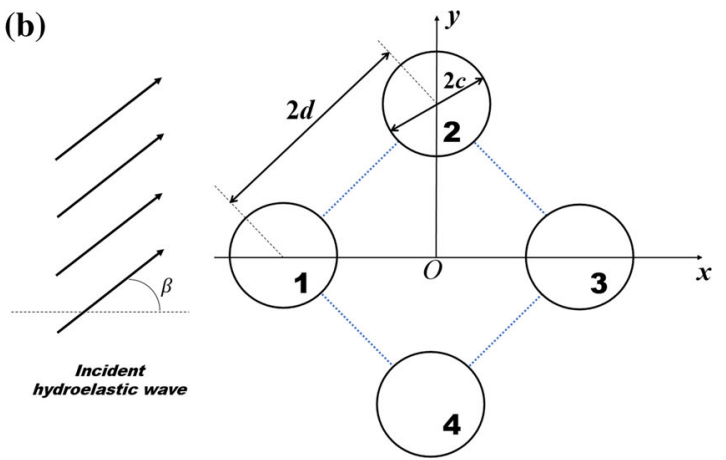

(b)
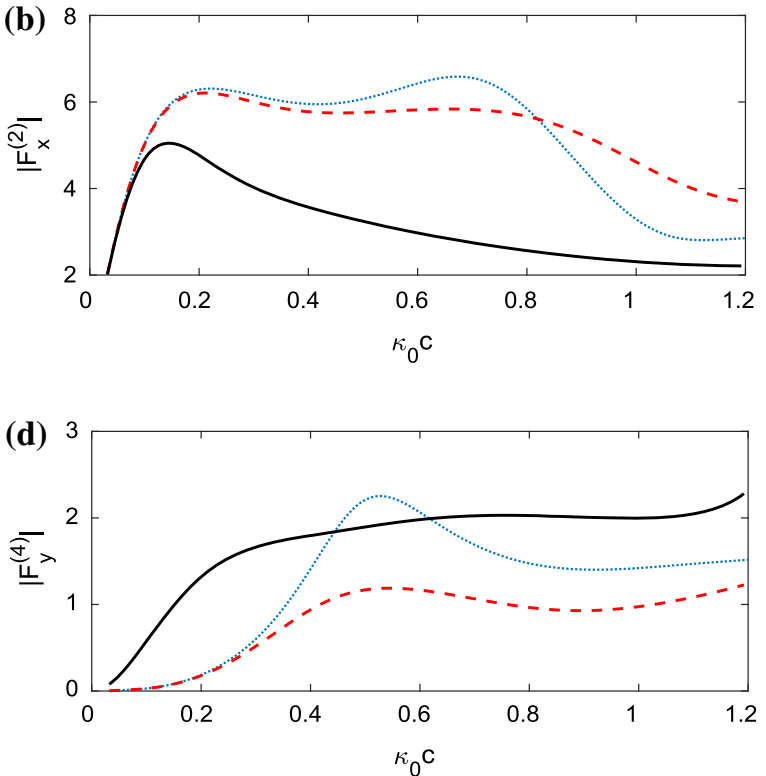

Fig. 5 Wave forces on cylinders shown in Fig. 4 with $\beta=0$ and $d / c=2$. Dashed lines: free edge; solid lines: clamped edge; dotted lines: open water $(h=0)\left(h=0.1, d=2, H=10, c_{j}=c=1, j=1,2,3,4, m_{\text {ice }}=0.09, L=4.5582\right)$

small $\kappa_{0}$, the diffraction effect is small, and therefore all the forces curves in Fig. 5a-c are very close to each other. For $F_{y}^{(4)}$ in Fig. 5d, the contribution from the incident wave is zero because of symmetry. The force is entirely due to diffraction, and therefore the difference between the free and clamped edge cases is visible even at small $\kappa_{0}$. As $\kappa_{0}$ increases, the diffraction effect becomes important for all the forces. This effect will be different for different ice sheet properties together with the edge condition.

We further investigate the difference between the cases in the open water and in the water covered by an ice sheet. At a given $\kappa_{0}$, we shall obtain different $\omega$ from Eq. (13) in these two cases. Due to the fourth power, the term $L \kappa_{0}^{4}$ increases with $\kappa_{0}$ very quickly, and its effect on $\omega$ becomes more obvious. For other $\kappa_{n}$ with $n \neq 0$, the difference in the two cases is further caused by the term $L \kappa_{n}^{4}-m_{\text {ice }} \omega^{2}$ in the case with ice sheet. This leads to two complex eigenvalues $\kappa_{-2}$ and $\kappa_{-1}$ magnitudes of which are infinite and effects of which do not exist in the case of open water. Also, when $n$ increases, the effect of the thickness of ice sheet, $h$, becomes more obvious, in particular at larger $\kappa_{0}$. It is therefore not surprising to see when $\kappa_{0}$ becomes larger, the effect of $h$ becomes more significant even when $L$ itself may be quite small. Another reason for the difference in the solutions of Eq. (21) is that the eigenfunctions in the ice-covered case is non-orthogonal, leading to the off-diagonal terms in the matrix equations. This is further complicated by the line integral term along the ice edge, contribution of which is different for the free edge and clamped edge cases. 

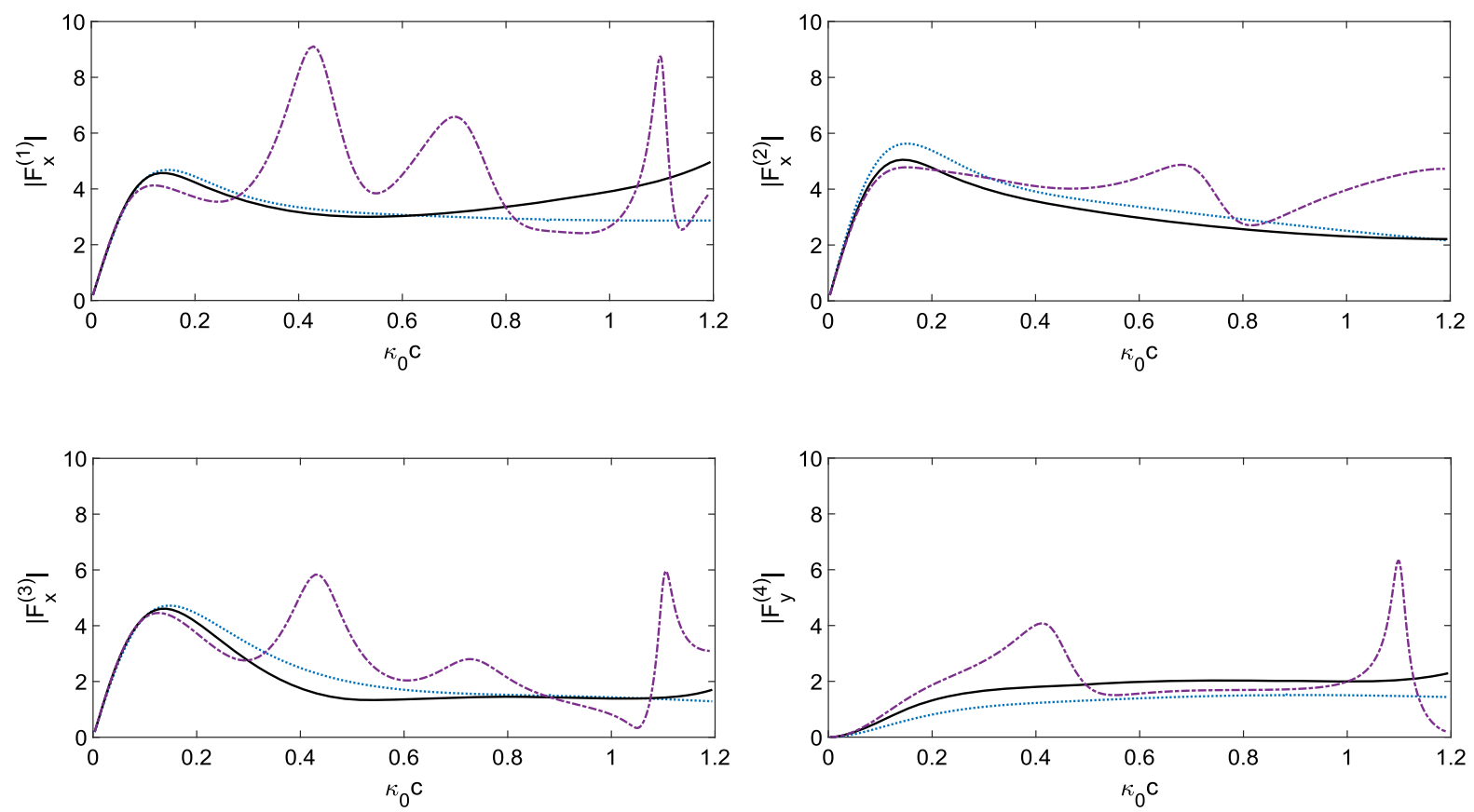

Fig. 6 Wave forces on cylinders in Fig. 4 for clamped edge case with $\beta=0$. Dotted lines: $d / c=1.25$; solid lines: $d / c=2.00$; dashed-dotted lines: $d / c=4.00\left(h=0.1, H=10, c_{j}=c=1, j=1,2,3,4, m_{\text {ice }}=0.09, L=4.5582\right)$

Results at a smaller and larger $d / c$, or $d / c=1.25$ and 4, for clamped edge case are provided in Fig. 6 to compare with the case for $d / c=2$. We can find that the curves become more oscillatory with $d / c$ becoming larger. Generally, the oscillation period is related to $\kappa_{0} d$ for this type of interaction problem, for example, as observed in a related problem (e.g. Li et al. [27]). Thus, when the results are plotted against $\kappa_{0} c$, the curve is more oscillatory for a larger $d$.

We further consider cases of different wave directions, with $\beta=\pi / 6$ and $\pi / 4$, respectively. The horizontal total wave forces, or $F_{h}^{(i)}=\sqrt{\left(F_{x}^{(i)}\right)^{2}+\left(F_{y}^{(i)}\right)^{2}}$, for clamped edge case are provided in Fig. 7 to compare with the results of $\beta=0$. From the figure, we can see that the magnitude of the force is not very much affected by the direction of the wave at small $\kappa_{0} c$. This is once again because the force is dominated by the incident potential at small $\kappa_{0} c$, magnitude of which is independent to the wave direction. For force on the second cylinder, it generally tends to decrease with the increase of $\beta$ for most of the range of wavenumber in the figure, while the tendency is opposite for other three cylinders.

The total vertical shear forces $\left|Q_{\text {tot }}^{(k)}\right|$ on each of these four cylinders are shown in Fig. 8. Due to symmetry, the results on the 2nd cylinder and the 4th cylinder are the same, and thus only that on the 2nd cylinder is displayed. From this figure and Fig. 5, we can find that the vertical shear force on each cylinder is much larger than the horizontal force. The vertical shear force on the 1st cylinder is larger than those on the 2nd and 3rd cylinders within the range of the wavenumber in the figures. It can be seen, when $\kappa_{0} c$ is small, the vertical shear forces on all cylinder are close. However, the forces are smaller than that on the cylinder in isolation.

\subsection{A single array of cylinders}

We then consider a single array of cylinders piercing through the ice sheet. All these cylinders are assumed to have the same radius $c$ and be equally placed along the $x$-axis in the global Cartesian coordinate system. The distance 

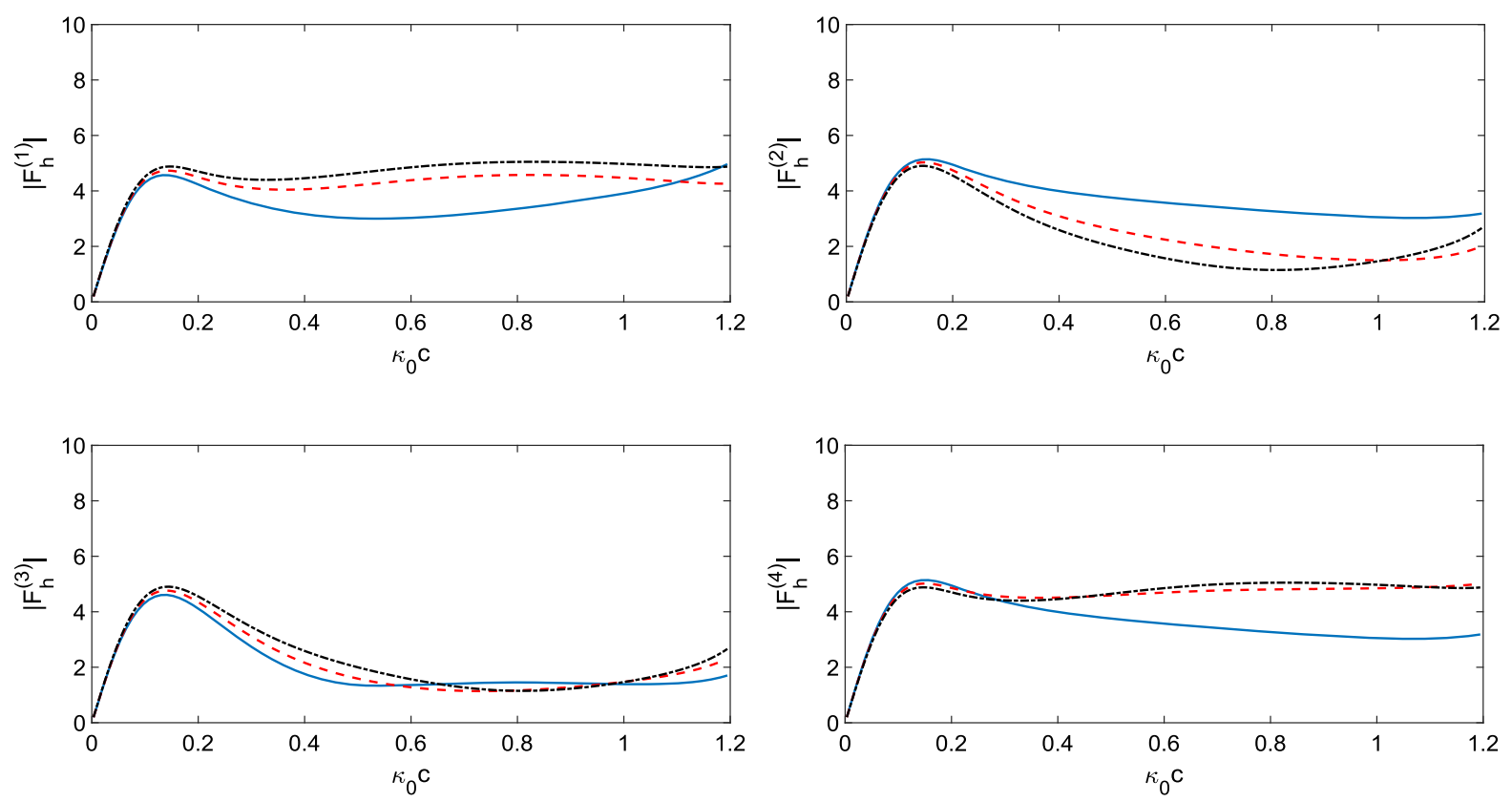

Fig. 7 Wave forces on cylinders in Fig. 4 with $d / c=2$. Solid lines: $\beta=0$; dashed lines: $\beta=\pi / 6$; dashed dotted lines: $\beta=\pi / 4$ $\left(h=0.1, m_{\text {ice }}=0.09, L=4.5582\right)$

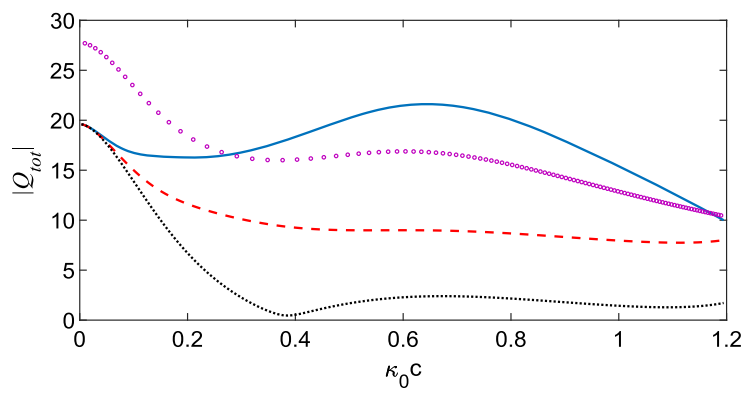

Fig. 8 Vertical shear forces on cylinders shown in Fig. 4 with $\beta=0$ and $d / c=2$. Solid line: on the 1st cylinder; dashed line: on the 2nd cylinder; dotted line: on the 3rd cylinder; circles: on a single cylinder. $\left(h=0.1, d=2, H=10, c_{j}=c=1, j=1,2,3,4\right.$, $\left.m_{\text {ice }}=0.09, L=4.5582\right)$
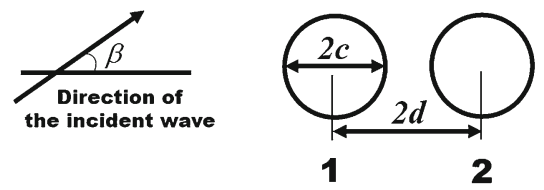

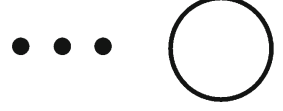

NC-2

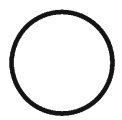

NC-1

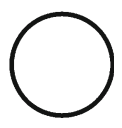

NC

Fig. 9 Sketch of a single array of cylinders in incident hydroelastic waves

between two axes of adjacent cylinders is $2 d$. The origin of the global system is set on the centre of the first cylinder (Fig. 9).

Simulations are made using two different edge conditions. The wave forces on cylinders in ice sheet with different thicknesses $h$ are obtained. Results for the open water problem are also provided for comparison. We first consider a single array of nine cylinders in the head wave, or $\beta=0$, and show the force along $x$-axis on the middle cylinder with respect to the wavenumber in Fig. 10, while the force in the $y$ direction is zero due to the symmetry. From the figure, we can find that the local peaks in the curves for open water problem observed in Fig. 2 also appear in the 


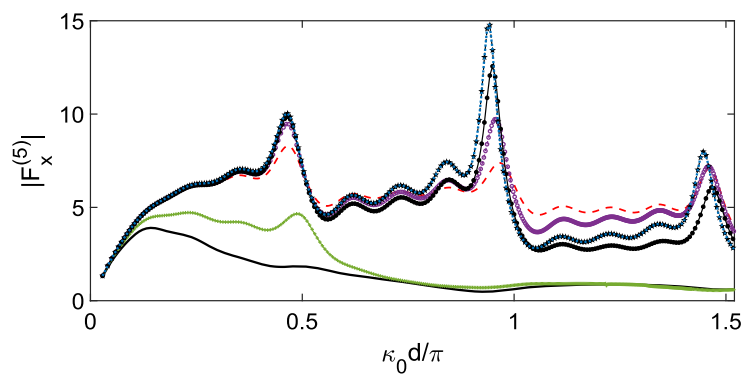

Fig. 10 Wave force on the middle body of a single array of 9 cylinders. Dashed lines: free edge cases (Dashed lines: $h=0.1$, $m_{\text {ice }}=0.09, L=4.5582$; dashed lines with open circle: $h=0.05, m_{\text {ice }}=0.045, L=0.5698$; dashed lines with filled star: $h=0.001$, $\left.m_{\text {ice }}=0.0009, L=4.5582 \times 10^{-6}\right)$. Solid lines: clamped edge cases (solid lines: $h=0.1, m_{\text {ice }}=0.09, L=4.5582$; solid lines with plus: $h=0.05, m_{\text {ice }}=0.045, L=0.5698$; solid lines with filled circle: $h=0.001, m_{\text {ice }}=0.0009, L=4.5582 \times 10^{-6}$ ). Dotted lines: open water case. $(\beta=0, c=1, d=4, H=10)$

curves of the free edge case. However, for clamped edge case, there are no obvious peaks in the case of $h=0.1$ and at $h=0.05$, and there is a milder peak around $\kappa_{0} d / \pi=0.5$ and no visible one near $\kappa_{0} d / \pi=1.0$. This shows that for this configuration, ice sheet can suppress this kind of interaction behaviour in the open water mentioned in Maniar and Newman [16], especially in the clamped cases. Mathematically, when $h \rightarrow 0$, the eigenvalues $\kappa_{n} \quad(n=0,1,2, \ldots)$ tend to those in open water and the eigenfunctions become orthogonal. Also, the complex roots $\kappa_{-1}$ and $\kappa_{-2}$, which do not exist in open water case, will tend to infinity as $h \rightarrow 0$. In such a case, Eq. (13) can be written as

$L \kappa_{n}^{4}+\rho g-m_{\text {ice }} \omega^{2}=\frac{\rho \omega^{2}}{\kappa_{n} \tanh \kappa_{n} H} \approx 0, n=-1$ or -2.

Thus, $\kappa_{-1}$ and $\kappa_{-2}$ can be obtained very easily as $\sqrt[4]{\left(\rho g-m_{\text {ice }} \omega^{2}\right) / L} \times \mathrm{e}^{\mathrm{i} \frac{\pi}{4}}$ and $\sqrt[4]{\left(\rho g-m_{\text {ice }} \omega^{2}\right) / L} \times \mathrm{e}^{\mathrm{i} \frac{3 \pi}{4}}$, as $L \rightarrow 0$. For other $\kappa_{n}(n \geq 0)$, the term associated with $L$ can be ignored in Eq. (13) as $L \rightarrow 0$; therefore, the eigenvalues will tend to those in open water.

When we replace the numerator of $\mathrm{X}\left(\kappa_{n}, \kappa_{n^{\prime}}\right)$ for $n \neq n^{\prime}\left(n, n^{\prime}=0,1,2 \ldots\right)$ with the right-hand side of Eq. (13), we have $\mathrm{X}\left(\kappa_{n}, \kappa_{n^{\prime}}\right)=-\frac{L}{\rho \omega^{2}}\left(\kappa_{n}^{2}+\kappa_{n^{\prime}}^{2}\right)\left(\kappa_{n} \times \tanh \kappa_{n} H\right)\left(\kappa_{n^{\prime}} \times \tanh \kappa_{n^{\prime}} H\right)$. This shows that $X$ term is proportional to $L$, which tends to zero as $h \rightarrow 0$. For the complex roots, $\kappa_{-1}$ and $\kappa_{-2}$, they tend to infinity as $L \rightarrow 0$. By taking limits in the equation of $\mathrm{X}\left(\kappa_{n}, \kappa_{n^{\prime}}\right)$ defined below Eq. (21), $\mathrm{X}\left(\kappa_{n}, \kappa_{n^{\prime}}\right) \rightarrow \frac{1}{\kappa_{n}+\kappa_{n^{\prime}}} \rightarrow 0$. In Eq. (21), for the $X$ term, it becomes orthogonal as $h \rightarrow 0$, or all the $n$ terms become independent. While for the terms associated with $L$, due to the line integral, it tends to zero apart from for $n=-2, n^{\prime}=-1$ or $n=-1, n^{\prime}=-2$. We also notice that the right-hand side of Eq. (21) is non-zero only when $n^{\prime}=0$. Thus, $a_{n m}$ is non-zero only when $n=0$, and only the term of $n=0$ needs to be kept in Eq. (25). This means when $h \rightarrow 0$, the force tends to that in the open water. To verify this, we have run a further simulation with $h=0.001$. Its results are much closer to those in the open water, and the typical features in the open water reappear.

The wave forces on cylinders with odd number are plotted in Fig. 11 to show their changes from cylinder to cylinder. We can see that for free edge case, the forces on these five cylinders have local peaks, and the peaks of cylinders near the centre are higher than those on the side. Between peaks the forces on upstream cylinders are quite oscillatory while those on downstream cylinders are much smoother. For clamped edge case, there is no obvious peak in the force curves. The largest wave force is on the first cylinder and the force generally decreases from the cylinder to cylinder in the wave direction.

A different wave direction with $\beta=\pi / 4$, is considered here. The horizontal wave forces on the middle cylinder against wavenumbers are shown in Fig. 12. We can find that compared with the zero-incident angle, local peaks in the curves of open water case as well as free edge case still exist around $\kappa_{0} d / \pi=0.5$ and 1.0, but with the peaks becoming less obvious and the position of the peak has moved to the right. 
Fig. 11 Wave forces changing from cylinder to cylinder. ( $\beta=0, h=0.1$, $c=1, d=4, H=10$, $\left.m_{\text {ice }}=0.09, L=4.5582\right)$.

a Free-edge case, $\mathbf{b}$ clamped-edge case
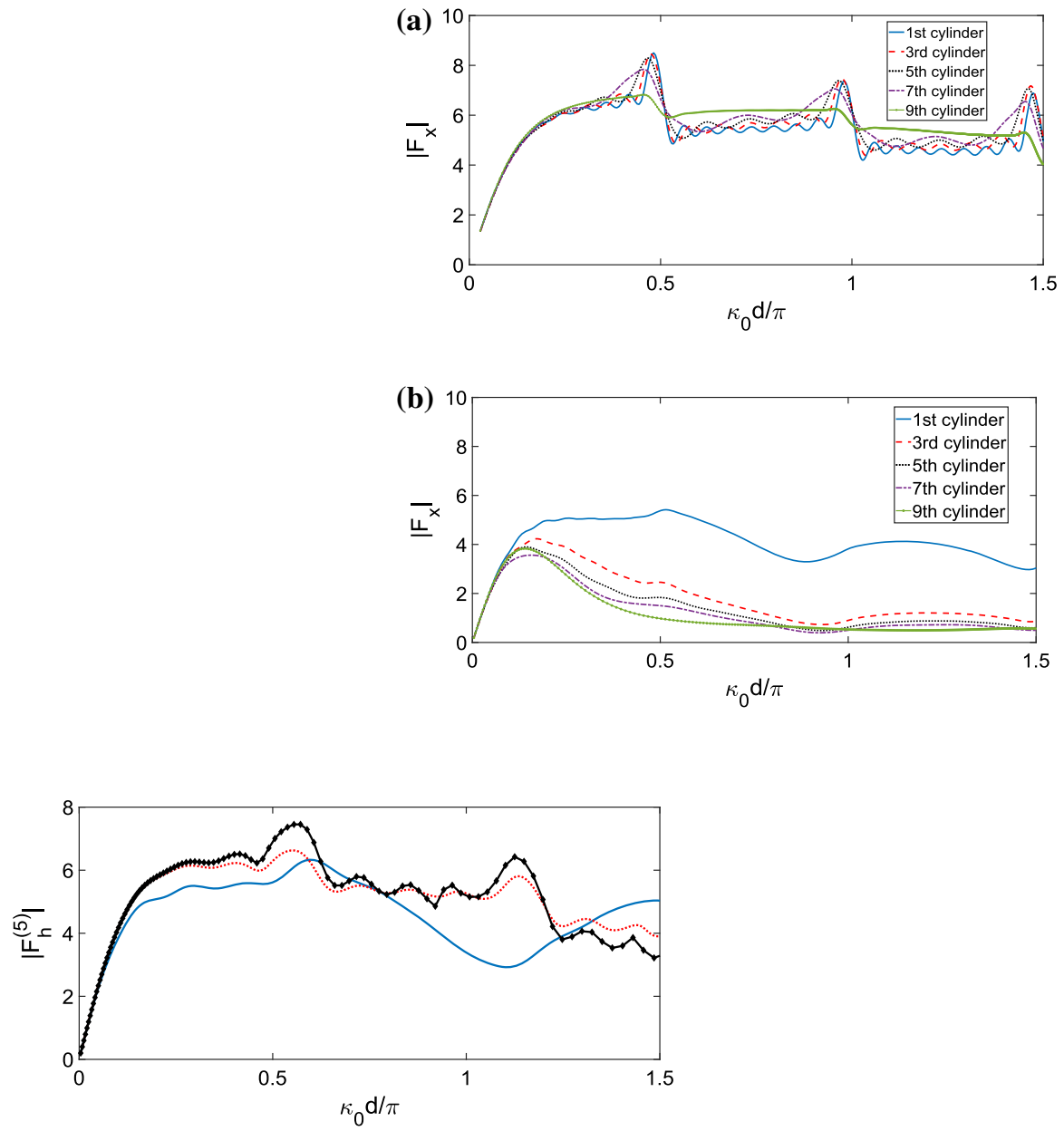

Fig. 12 Horizontal wave force on the middle body of a single array of nine cylinders with $\beta=\pi / 4$. Dotted lines: free edge; solid lines: clamped edge; solid lines with filled diamond: open water case $\left(\beta=0, h=0.1, c=1, d=4, H=10, m_{\text {ice }}=0.09, L=4.5582\right)$

(a)

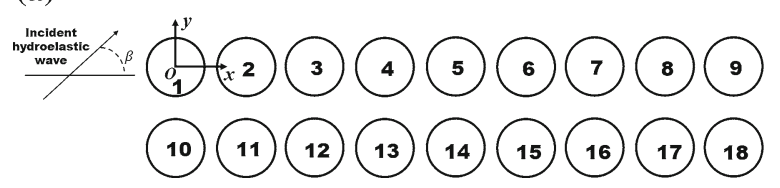

(b)

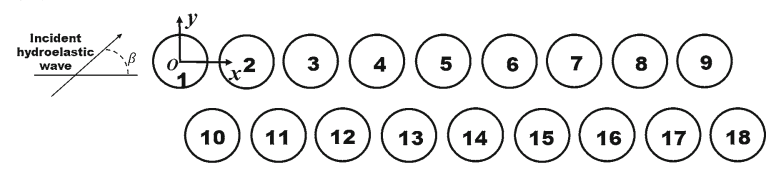

Fig. 13 Sketch of two arrays of cylinders $(N C=18)$

\subsection{Two arrays of cylinders in side by side arrangement}

The diffraction problem of a double-array of $2 \times 9$ cylinders arranged in Fig. 13a have been considered by Evans and Porter [40] based on the method of Linton and Evans [13] in open water and by Wang and Wu [41] based on a fully nonlinear finite element method in a numerical wave tank. In present work, we consider a similar problem in the context of ice-covered ocean with free/clamped edge conditions. The origin of the global coordinate system is still chosen at the centre of the first cylinder. The coordinates of the centres of the two arrays of cylinders are at $X_{j}=X_{j+9}=(j-1) \times 2 d(j=1, \ldots, 9)$ and $Y_{j}=Y_{j+9}+2 l=0(j=1, \ldots, 9)$, shown in Fig. 13a. $l$ is half 
(a)

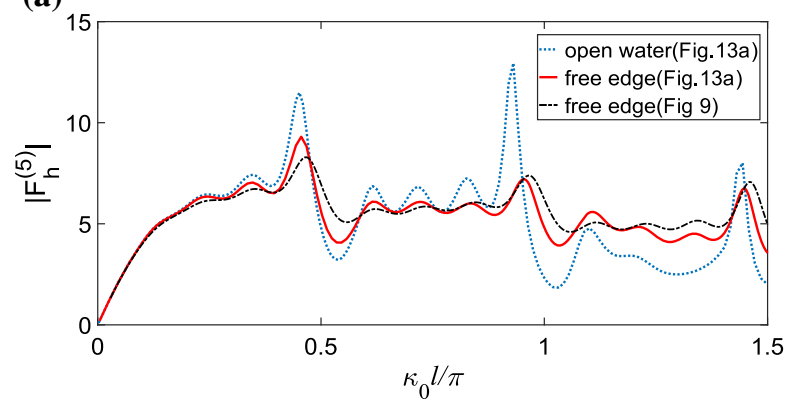

(b)

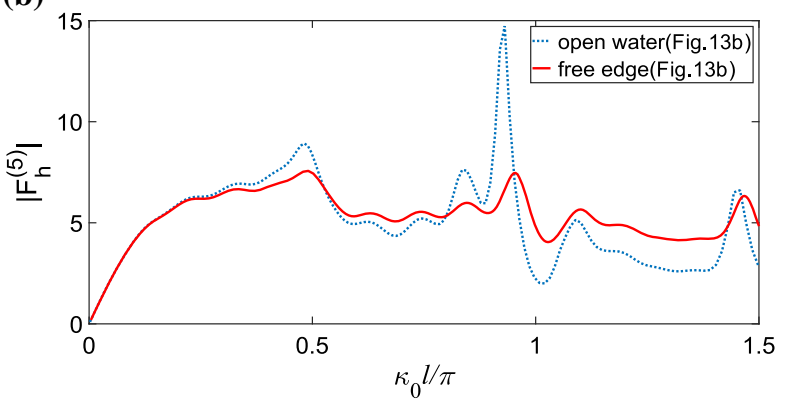

Fig. 14 Wave force on the 5th cylinder with free edge for different arrangements $(\beta=0, h=0.1, c=1, l=d=4, H=10)$. a Arrangement in Fig. 13a; b arrangement in Fig. 13b

Fig. 15 Total wave forces on the 5 th cylinder with clamped edge $(\beta=0, h=0.1, c=1$, $l=d=4, H=10)$

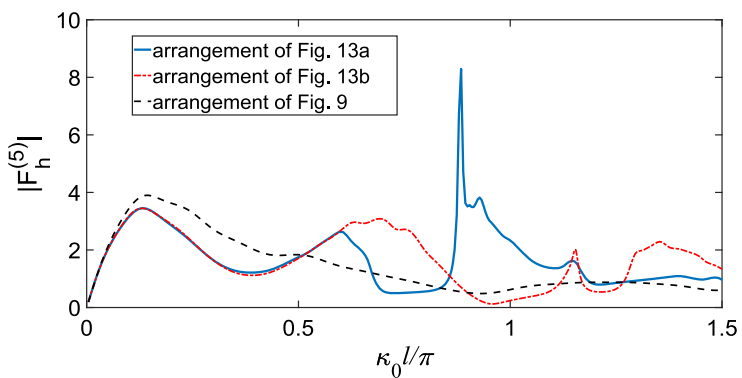

of the distance between the two arrays. In addition, we also consider a double-array of cylinders staggered with $X_{j}=X_{j+9}-d=(j-1) \times 2 d(j=1, \ldots, 9)$ and $Y_{j}=Y_{j+9}+2 l=0(j=1, \ldots, 9)$, as shown in Fig. $13 \mathrm{~b}$.

In the following wave, or $\beta=0$, the wave forces on the 5th cylinder for both two arrangements at free/clamped edge conditions against wavenumbers are, respectively, displayed in Figs. 14 and 15 to see how the different arrangements of the cylinders affect the results. The corresponding results in the cases of open water and the case of $1 \times 9$ cylinders shown in Fig. 9 are also displayed for comparison. For the free edge case shown in Fig. 14, the local peaks can be seen around the wavenumbers $\kappa_{0} d / \pi=0.5$ or 1.0 for the arrangement in Fig. 13a. Evans and Porter [40] reported that large forces can occur on the middle pair of cylinders in open water at certain wavenumbers or frequencies, which is also reflected in our results in open water shown in Fig. 14a. However, for the staggered arrangement in Fig. 13b, the first local peak becomes less obvious, and it has smaller influence on the second one.

For the clamped edge in Fig. 15, we can see a sharp local peak before $\kappa_{0} l / \pi=1.0$ in the curve of the total wave force on the 5th cylinder arranged in Fig. 13a. A similar phenomenon can also be found for the wave forces on other cylinder pairs near the middle position, such as on the 4th/13th and 6th/15th cylinders. In the double-array case, each array of cylinders can be approximately treated as a wall if the gap between two neighbouring cylinders is small. The flow between the two arrays of cylinders will be similar to that in a narrow tank. This is similar to that of a circular array of cylinders considered by Evans and Porter [18], where the inner flow resembles that in a circular tank. The difference between these two cases is that when the cylinders are in a circular arrangement, the internal region is entirely cut off from the external one if the distance between two neighbouring cylinders becomes zero and there will be no motion in the internal region. In the case of two arrays, the flow between the two arrays will still exist because of the openings at the two ends even when the distance is zero. The tank resonance effect remains. The result in Fig. 14 for the arrangement of cylinders in Fig. 13 reflects the resonance of the long tank. It ought to be pointed out that in the case of the long straight tank, $l$ is the half distance between two tank walls. Here $l$ is the half distance between the centre lines of the two arrays. On the other hand, we should note that even though when $d \rightarrow c$, it is not a straight tank wall, but curved. The distance between the two walls varies between $2 l$ and $2 l-2 c$. Thus, the approximation of two straight tank walls for the two arrays of cylinders should be made in the 
Fig. 16 Total wave forces on the middle cylinder with clamped edge at different column numbers $(\beta=0, h=0.1, c=1$, $l=d=4, H=10)$

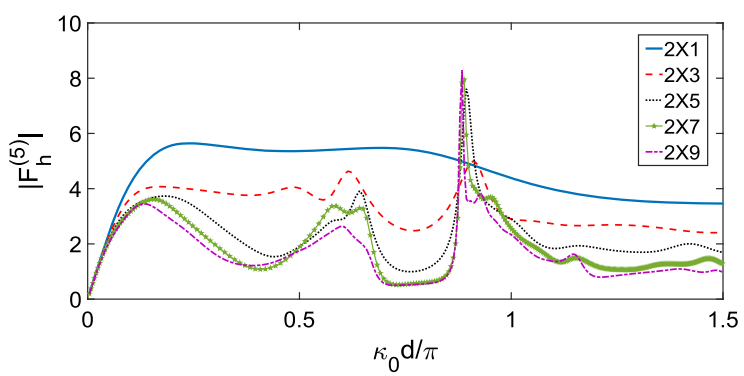

context of $l \gg c$. Furthermore, the distance between the two neighbouring cylinders should be small. Therefore, the analogy to the straight tank wall could be used only qualitatively but not quantitatively.

We further consider how the total horizontal forces on middle cylinder with $m$, when there is a double array of $2 \times m(m=1,3,5,7)$ cylinders with clamped edge condition. In the simulations, cylinders near the middle, such as 5th and 14th cylinders, are always kept and those on the sides will be added on as $m$ increases. Their results are displayed and compared in Fig. 16. From the figure we can see that the force curve for the case of $m=1$ is very flat and smooth. However, when $m$ increases $(m>3)$, we notice that sharp peaks start to occur around the same wavenumber. This suggests that the long tank effect has already appeared at relative small number of cylinders.

\section{Conclusions}

By means of eigenfunction expansions and the Green's second identity repeatedly for domains outside individual cylinders, solutions for interactions of hydroelastic waves with multiple vertical cylinders have been obtained in an efficient way. Different arrangements of cylinders have been considered, including cylinders arranged in square, in a single array, in a double array and in a staggered double array. The wave forces on the cylinders piercing through ice sheet under clamped and free edge conditions are obtained and analysed. Based on these results, we can draw the following conclusions:

(1) When ice thickness of the ice sheet $h \rightarrow 0$, the results for the hydroelastic wave will tend to those as for the free surface case in the open water.

(2) For vertical cylinders arranged in an array, we can find that local peaks around $\kappa_{0} d / \pi=0.5$ and 1.0 are shown in free edge case at relatively thicker ice sheet. It is not obvious in clamped edge case at similar ice sheet thickness, but will also be obvious at a far thinner ice sheet.

(3) For two arrays of cylinders arranged side by side and symmetric to $y=-d$, we can find that large forces can appear on the middle pairs for clamped edge case but not for the free edge. The 'enclosure effect', which means that each array of cylinders is approximated as a wall, can qualitatively account for such phenomenon. In staggered case, this approximate effect becomes less obvious.

Acknowledgements The first author is sponsored by the joint scholarship from the University College London and the China Scholarships Council, to both of which he is most grateful. The authors also gratefully acknowledge financial support from the Lloyd's Register Foundation (LRF) through the joint centre involving the University College London, the Shanghai Jiao Tong University, and the Harbin Engineering University. The LRF helps protect life and property by supporting engineering-related education, public engagement, and the application of research.

Open Access This article is distributed under the terms of the Creative Commons Attribution 4.0 International License (http:// creativecommons.org/licenses/by/4.0/), which permits unrestricted use, distribution, and reproduction in any medium, provided you give appropriate credit to the original author(s) and the source, provide a link to the Creative Commons license, and indicate if changes were made. 


\section{References}

1. Havelock TH (1940) The pressure of water waves upon a fixed obstacle. Proc R Soc Lond Ser A 175:409-421

2. MacCamy RC, Fuchs RA (1954) Wave forces on piles: a diffraction theory. US Army Corps of Engineers Beach Erosion Board. Tech. Memo No. 69

3. Chen HS, Mei CC (1971) Scattering and radiation of gravity waves by an elliptical cylinder. Rep. No. 140. Parsons Laboratory, Department of Civil Engineering, Massachusetts Institute of Technology, Cambridge

4. Williams AN (1985) Wave forces on an elliptic cylinder. J Waterw Port Coast Ocean Eng 111(2):433-449

5. Isaacson MDSQ (1978) Vertical cylinders of arbitrary section in waves. J Waterw Port Coast Ocean Eng 104(3):309-324

6. Wu XJ, Price WG (1991) Evaluation of wave drift forces on vertical cylinders of arbitrary geometry, with application to tension leg platforms (TLPs). Ocean Eng 18(1-2):1-15

7. Zhu S, Moule G (1994) Numerical calculation of forces induced by short-crested waves on a vertical cylinder of arbitrary crosssection. Ocean Eng 21(7):645-662

8. Liu J, Guo A, Li H (2016) Analytical solution for the linear wave diffraction by a uniform vertical cylinder with an arbitrary smooth cross-section. Ocean Eng 126:163-175

9. Mansour AM, Williams AN, Wang KH (2002) The diffraction of linear waves by a uniform vertical cylinder with cosine-type radial perturbations. Ocean Eng 29(3):239-259

10. Dişibüyük NB, Korobkin AA, Yilmaz O (2017) Linear wave interaction with a vertical cylinder of arbitrary cross section: an asymptotic approach. J Waterw Port Coast Ocean Eng 143(5):04017028

11. Ohkusu M (1974) Hydrodynamic forces on multiple cylinders in waves. In: Proceedings of international symposium on the dynamics of marine vehicles and structures in waves. Institution of Mechanical Engineers, London

12. Kagemoto H, Yue DK (1986) Interactions among multiple three-dimensional bodies in water waves: an exact algebraic method. J Fluid Mech 166:189-209

13. Linton CM, Evans DV (1990) The interaction of waves with arrays of vertical circular cylinders. J Fluid Mech 215:549-569

14. Eatock Taylor R, Chau FP (1992) Wave diffraction theory—some developments in linear and nonlinear theory. J Offshore Mech Arct Eng 114(3):185-194

15. Malenica Š, Eatock Taylor R, Huang JB (1999) Second-order water wave diffraction by an array of vertical cylinders. J Fluid Mech 390:349-373

16. Maniar HD, Newman JN (1997) Wave diffraction by a long array of cylinders. J Fluid Mech 339:309-330

17. Ursell F (1951) Trapping modes in the theory of surface waves. In: Mathematical proceedings of the Cambridge Philosophical Society, vol 47, No. 2. Cambridge University Press, Cambridge, pp 347-358

18. Evans DV, Porter R (1997) Near-trapping of waves by circular arrays of vertical cylinders. Appl Ocean Res 19(2):83-99

19. Duclos G, Clément AH (2004) Wave propagation through arrays of unevenly spaced vertical piles. Ocean Eng 31(13):1655-1668

20. Walker DAG, Eatock Taylor R (2005) Wave diffraction from linear arrays of cylinders. Ocean Eng 32(17):2053-2078

21. Wang CZ, Wu GX (2007) Time domain analysis of second-order wave diffraction by an array of vertical cylinders. J Fluids Struct 23(4):605-631

22. Sturova IV (2014) Wave generation by an oscillating submerged cylinder in the presence of a floating semi-infinite elastic plate. Fluid Dyn 49(4):504-514

23. Sturova IV (2015) Radiation of waves by a cylinder submerged in water with ice floe or polynya. J Fluid Mech 784:373-395

24. Sturova IV (2015) The effect of a crack in an ice sheet on the hydrodynamic characteristics of a submerged oscillating cylinder. J Appl Math Mech 79(2):170-178

25. Ren K, Wu GX, Thomas GA (2016) Wave excited motion of a body floating on water confined between two semi-infinite ice sheets. Phys Fluids 28(12):127101

26. Li ZF, Shi YY, Wu GX (2017) Large amplitude motions of a submerged circular cylinder in water with an ice cover. Eur J Mech B 65:141-159

27. Li ZF, Shi YY, Wu GX (2017) Interaction of wave with a body floating on a wide polynya. Phys Fluids 29(9):097104

28. Li ZF, Shi YY, Wu GX (2018) Interaction of waves with a body floating on polynya between two semi-infinite ice sheets. J Fluids Struct 78:86-108

29. Ren K, Wu GX, Ji CY (2018) Wave diffraction and radiation by a vertical circular cylinder standing in a three-dimensional polynya. J Fluids Struct 82:287-307

30. Brocklehurst P, Korobkin AA, Părău EI (2010) Interaction of hydro-elastic waves with a vertical wall. J Eng Math 68(3-4):215-231

31. Bhattacharjee J, Soares CG (2012) Flexural gravity wave over a floating ice sheet near a vertical wall. J Eng Math 75(1):29-48

32. Malenica Š, Korobkin AA, Gueret R (2003) Water wave diffraction by vertical circular cylinder in partially frozen sea. In: The 18th International workshop on water waves and floating bodies, Le Croisic, France

33. Brocklehurst P, Korobkin A, Părău EI (2011) Hydroelastic wave diffraction by a vertical cylinder. Philos Trans R Soc Lond A 369(1947):2832-2851

34. Korobkin AA, Malenica S, Khabakhpasheva T (2018) Interaction of hydroelastic waves in ice cover with vertical walls. In: The 33rd international workshop on water waves and floating bodies, Guidel-Plages, France

35. Fox C, Squire VA (1994) On the oblique reflexion and transmission of ocean waves at shore fast sea ice. Philos Trans R Soc Lond A 347:185-218 
36. Timoshenko S, Woinowsky-Krieger S (1959) Theory of plates and shells. McGraw-Hill, Singapore

37. Abramowitz M, Stegun IA (1965) Handbook of mathematical functions with formulas, graphs, and mathematical table, vol 2172. Dover, New York

38. Sahoo T, Yip TL, Chwang AT (2001) Scattering of surface waves by a semi-infinite floating elastic plate. Phys Fluids 13(11):32153222

39. Ugural A (1981) Stresses in plates and shells. McGraw Hill, New York

40. Evans DV, Porter R (1997) Trapped modes about multiple cylinders in a channel. J Fluids Mech 339:331-356

41. Wang CZ, Wu GX (2010) Interactions between fully nonlinear water waves and cylinder arrays in a wave tank. Ocean Eng 37(4):400-417 